\title{
Supporting spectra
}

\section{An improved method for the synthesis of nucleoside triphosphate analogues}

\author{
Samy Mohamady ${ }^{\ddagger}$ and David L. Jakeman ${ }^{\dagger *}$ \\ ${ }^{\dagger}$ College of Pharmacy, Dalhousie University, 5968 College ST. Halifax, Nova Scotia, B3H 3J5, Canada. \\ ${ }^{\ddagger}$ Department of Chemistry, Dalhousie University, Halifax, Nova Scotia, B3H 3J3, Canada. \\ david.jakeman@dal.ca
}

NMR spectra for the following compounds:

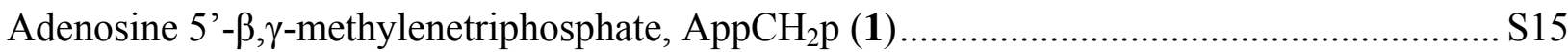

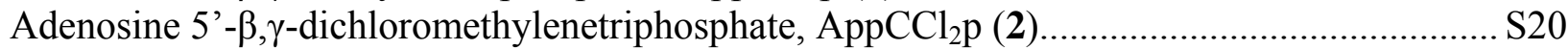

Adenosine 5'- $\beta, \gamma$-fluoromethylenetriphosphate, AppCHFp (3) ......................................... S25

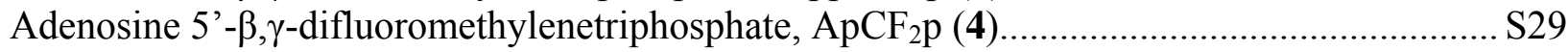

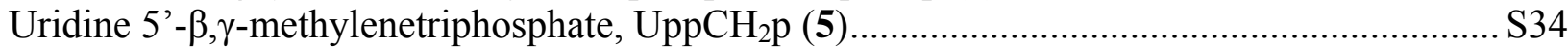

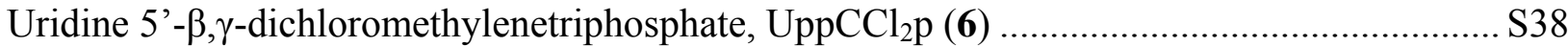

Uridine 5 '- $\beta, \gamma$-fluoromethylenetriphosphate, UppCHFp (7) .............................................. S42

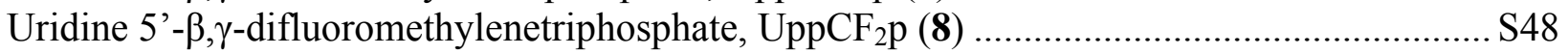




\section{Adenosine 5'- $\beta, \gamma-$-methylenetriphosphate, $\mathrm{AppCH}_{2} \mathrm{p}$ (1)}

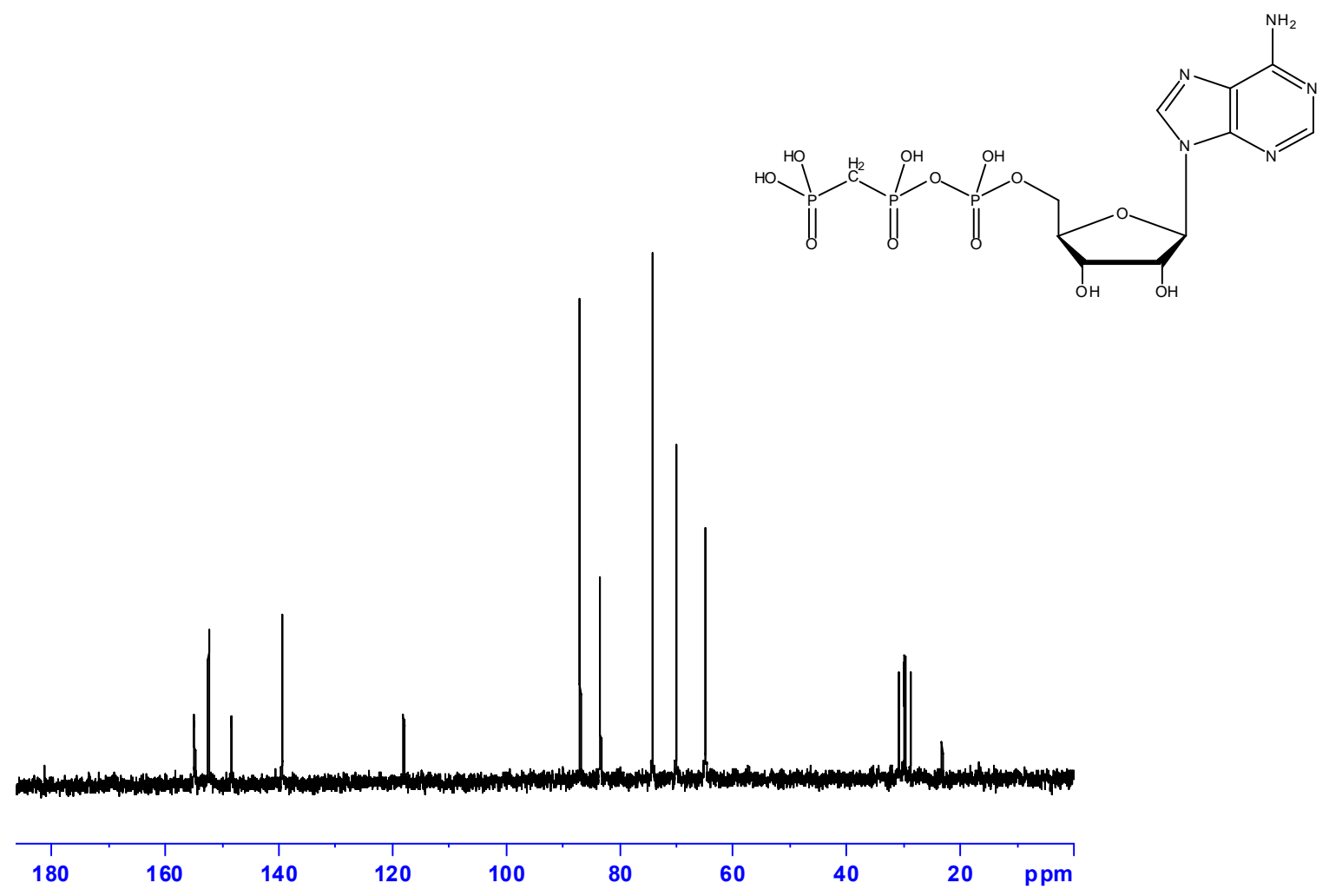

$\mathrm{CH}_{2 \beta \gamma}$

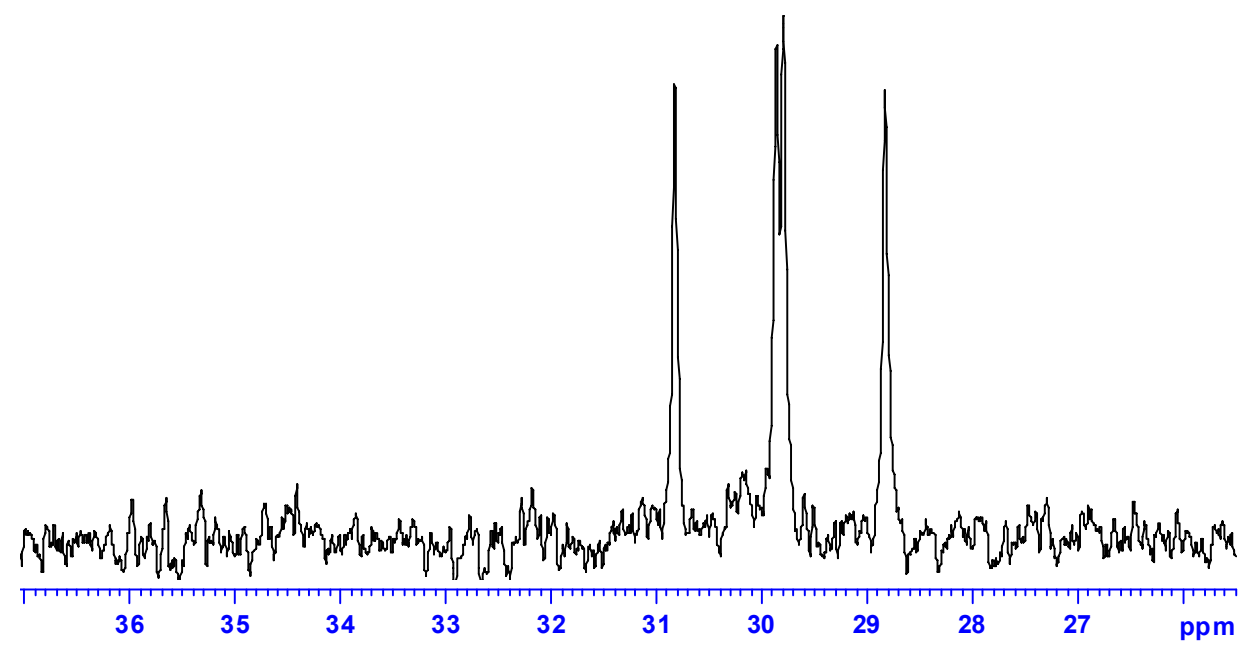


Groups Jakeman

Phosphorylmethyl ADP

WATER D20 $\{\mathrm{C}: \backslash \mathrm{nmr}$ _users $\}$ mohamady 7

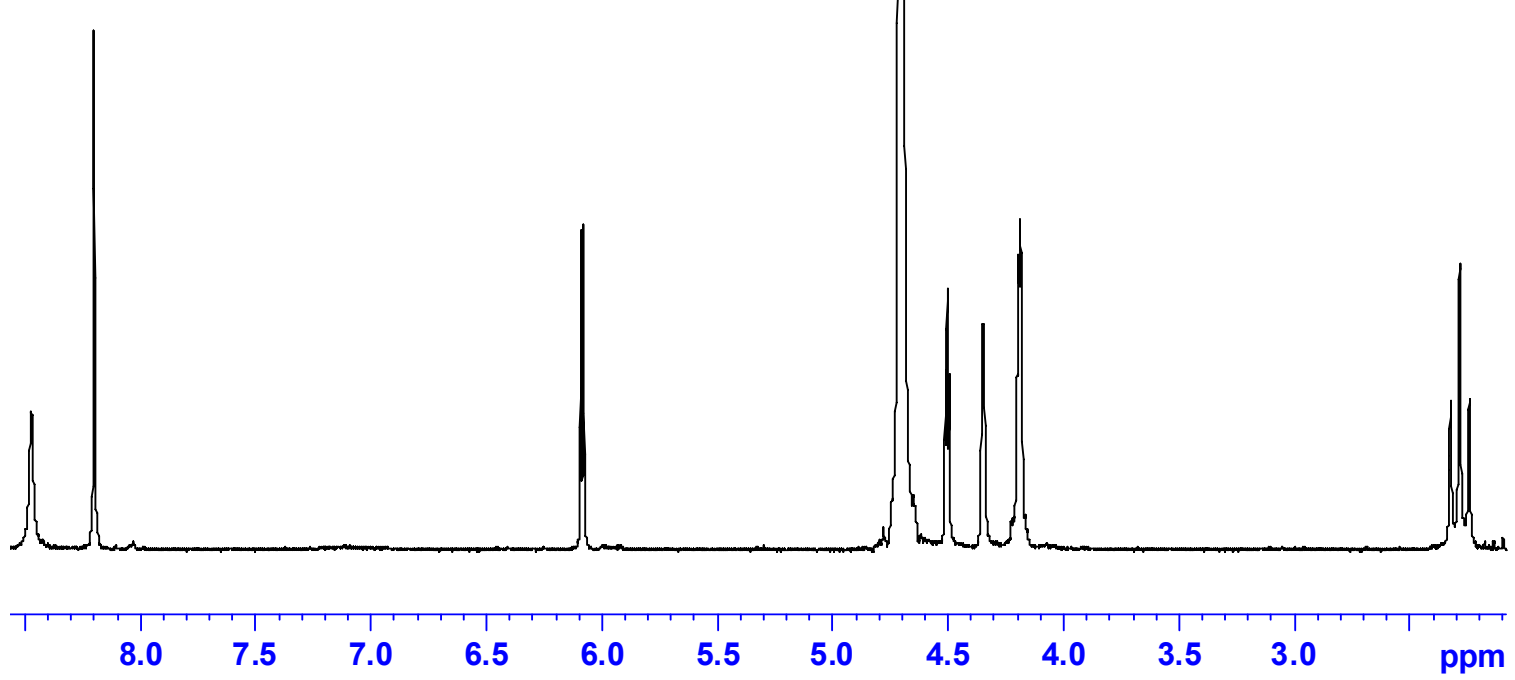




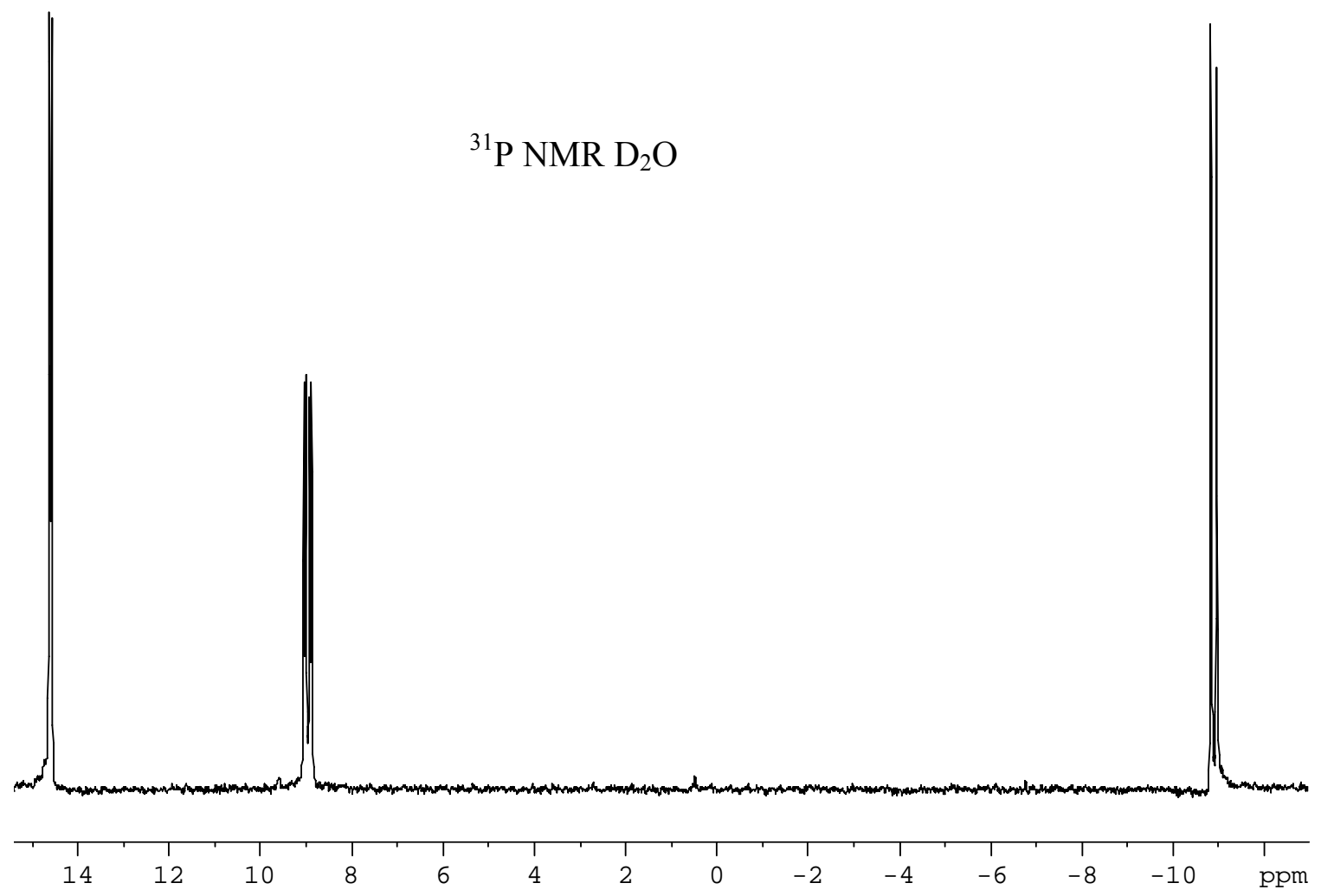


2d_CoSY D20 \{C:\nmr_users $\}$ mohamady 6

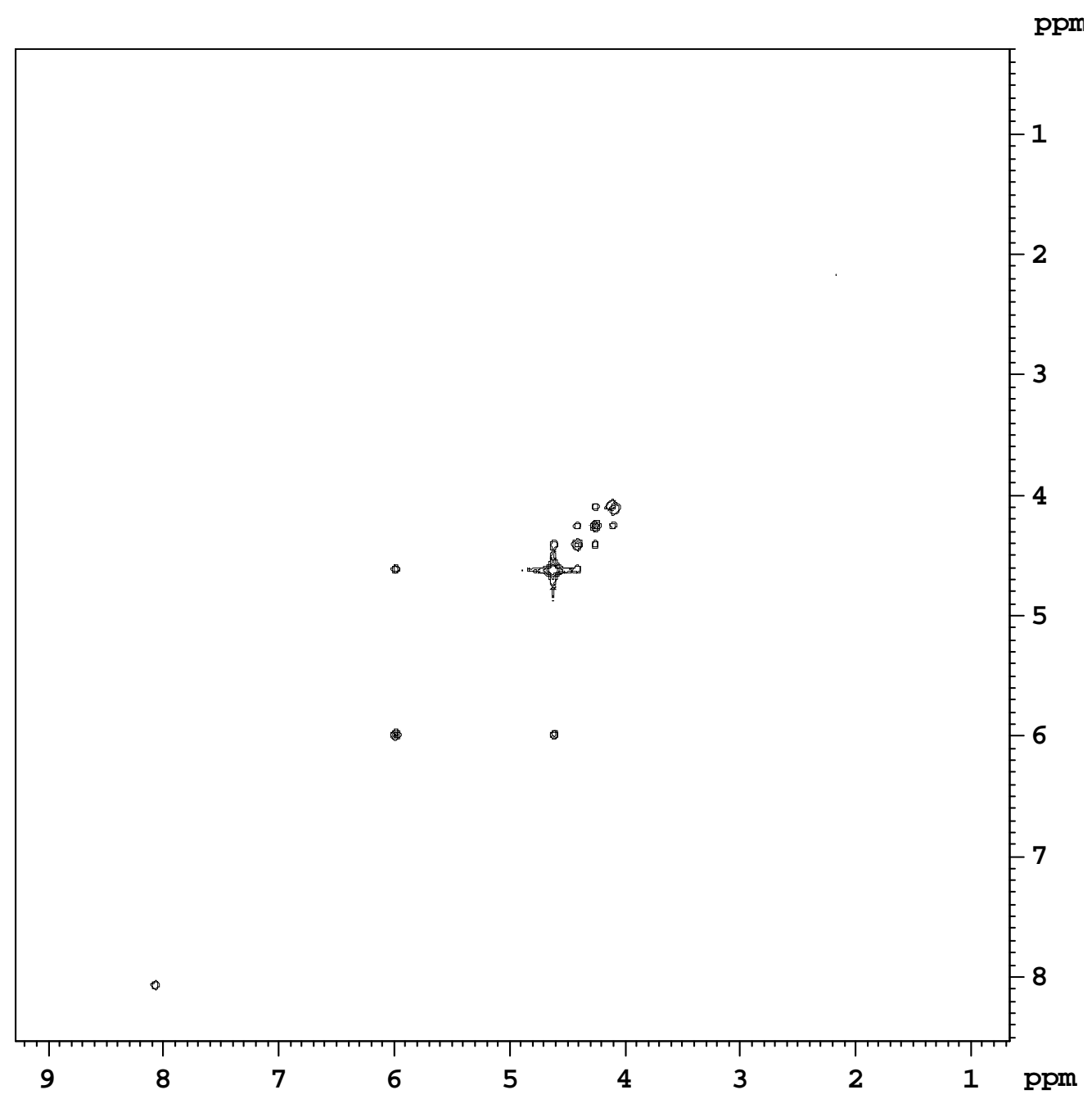


Phosphoryl methyl ADP 2d_HSQC D20 \{C: \nmr_users\} mohamady 6

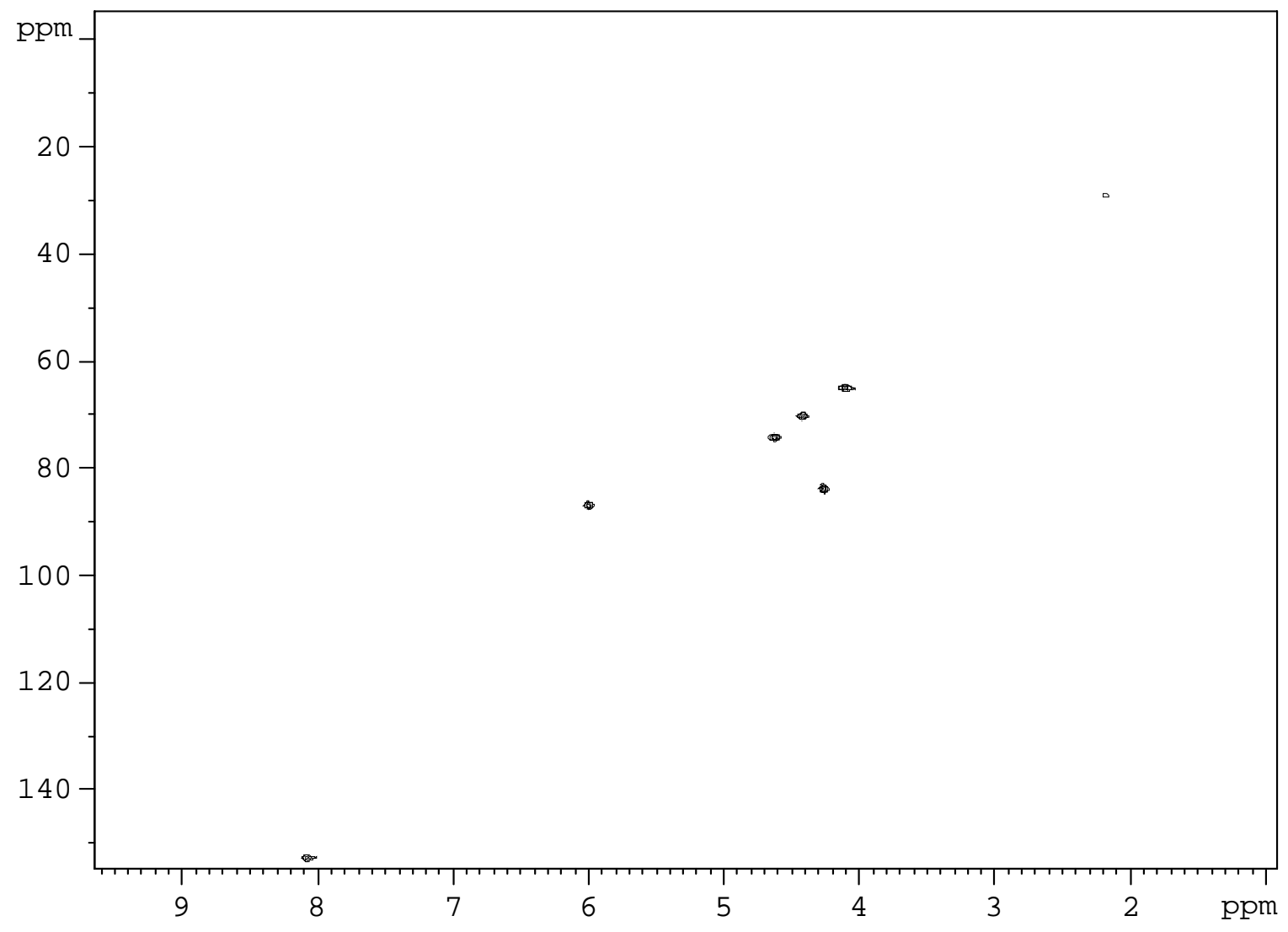




\section{Adenosine 5'- $\beta$,Y-dichloromethylenetriphosphate, $A p p C \mathrm{Cl}_{2} p$}

(2)

Phosphoryldichloromethyl ADP
1d_carbon_narrow D20 \{C:\nmr_users\} mohamady 5
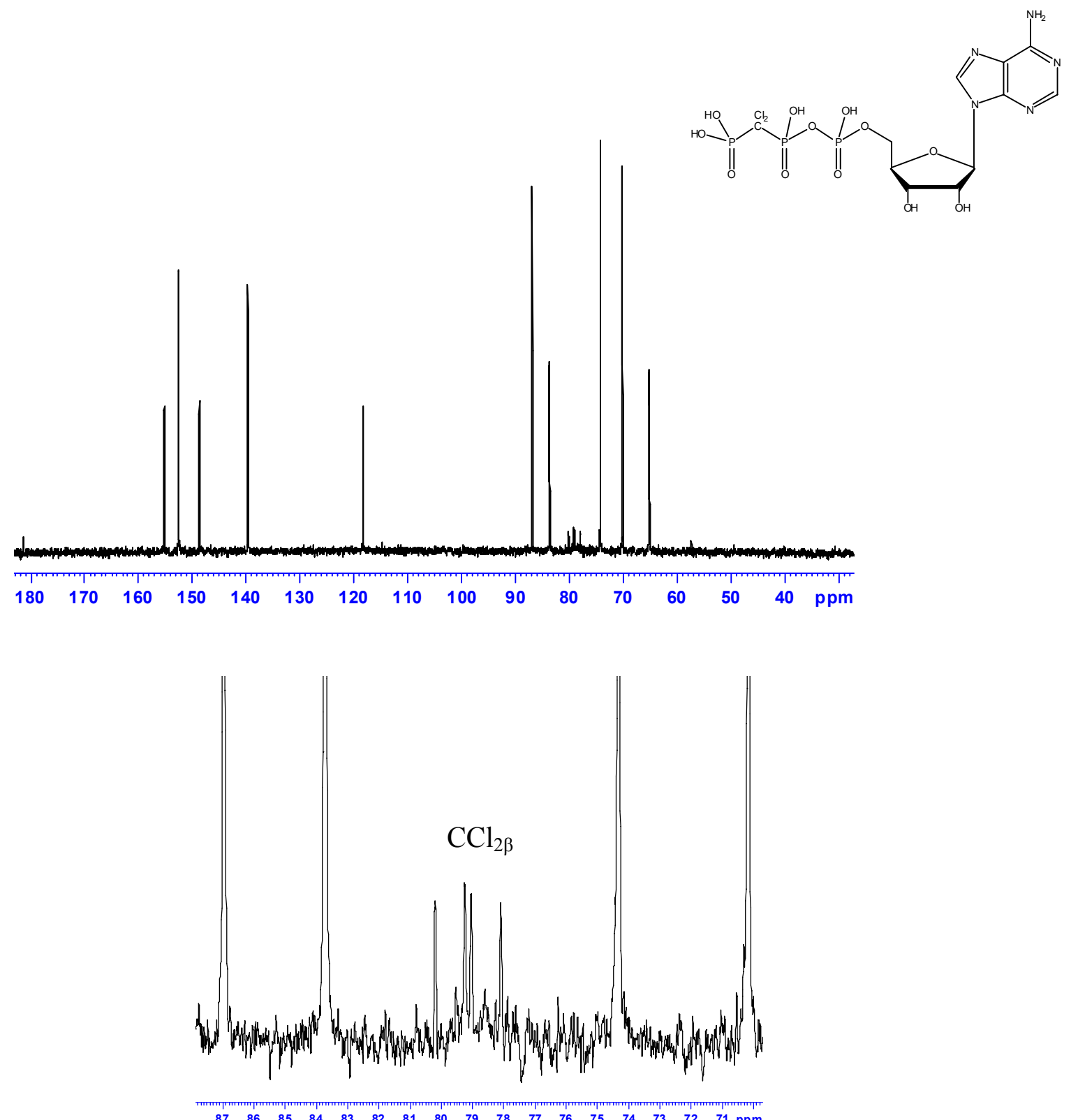


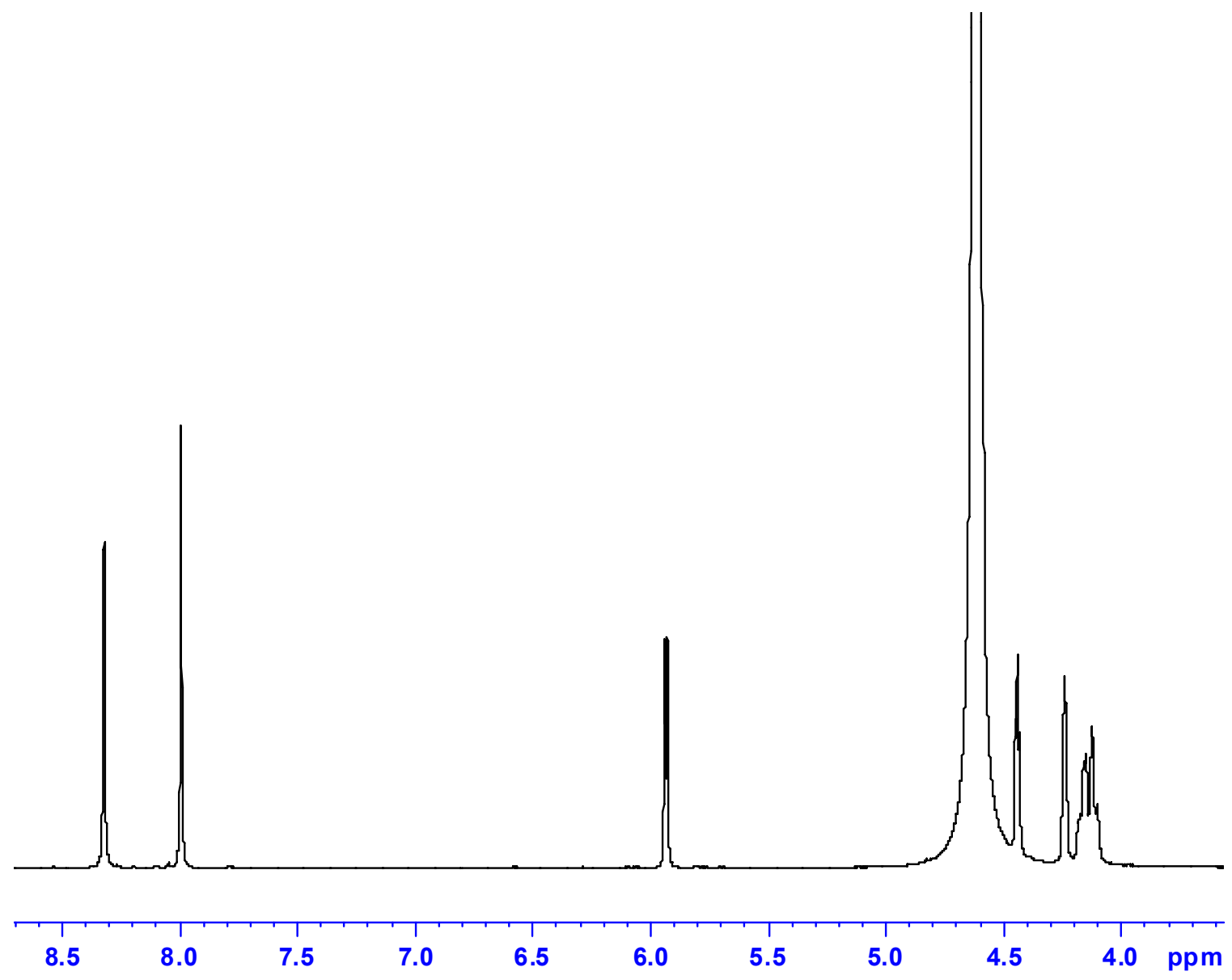




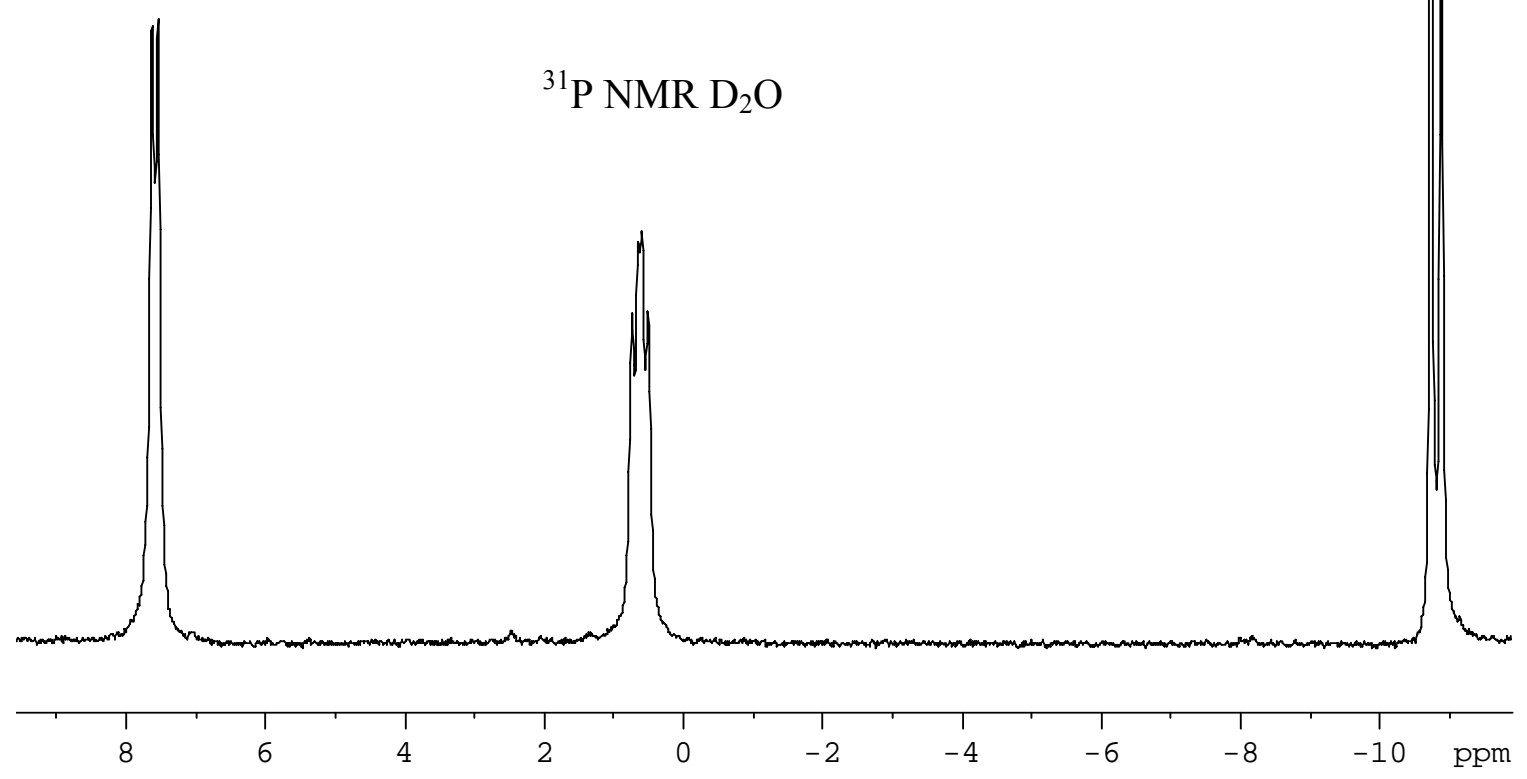




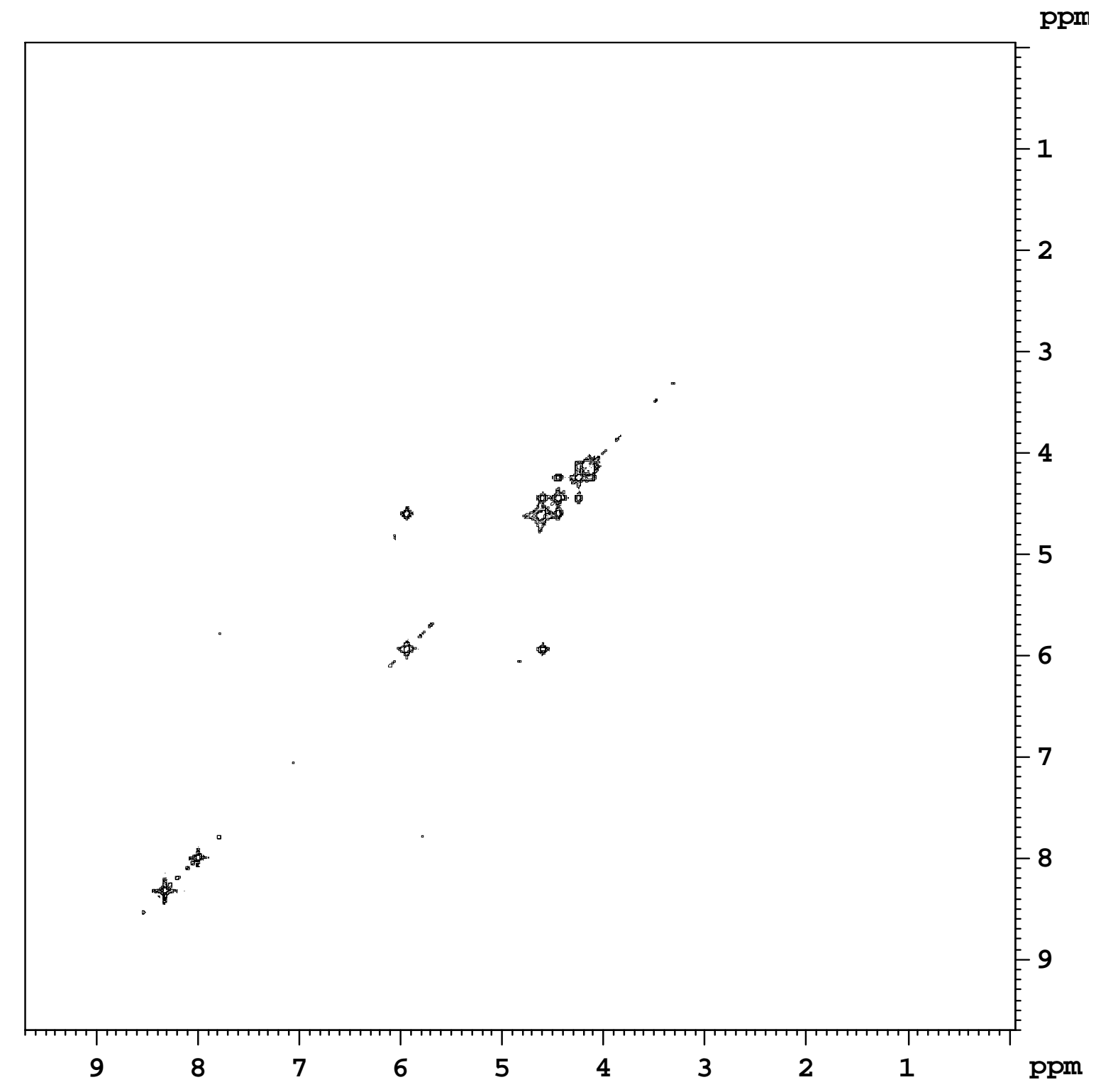


Phosphoryl dichloromethyl ADP

2d_HSQC D20 \{C: \nmr_users\} mohamady 4

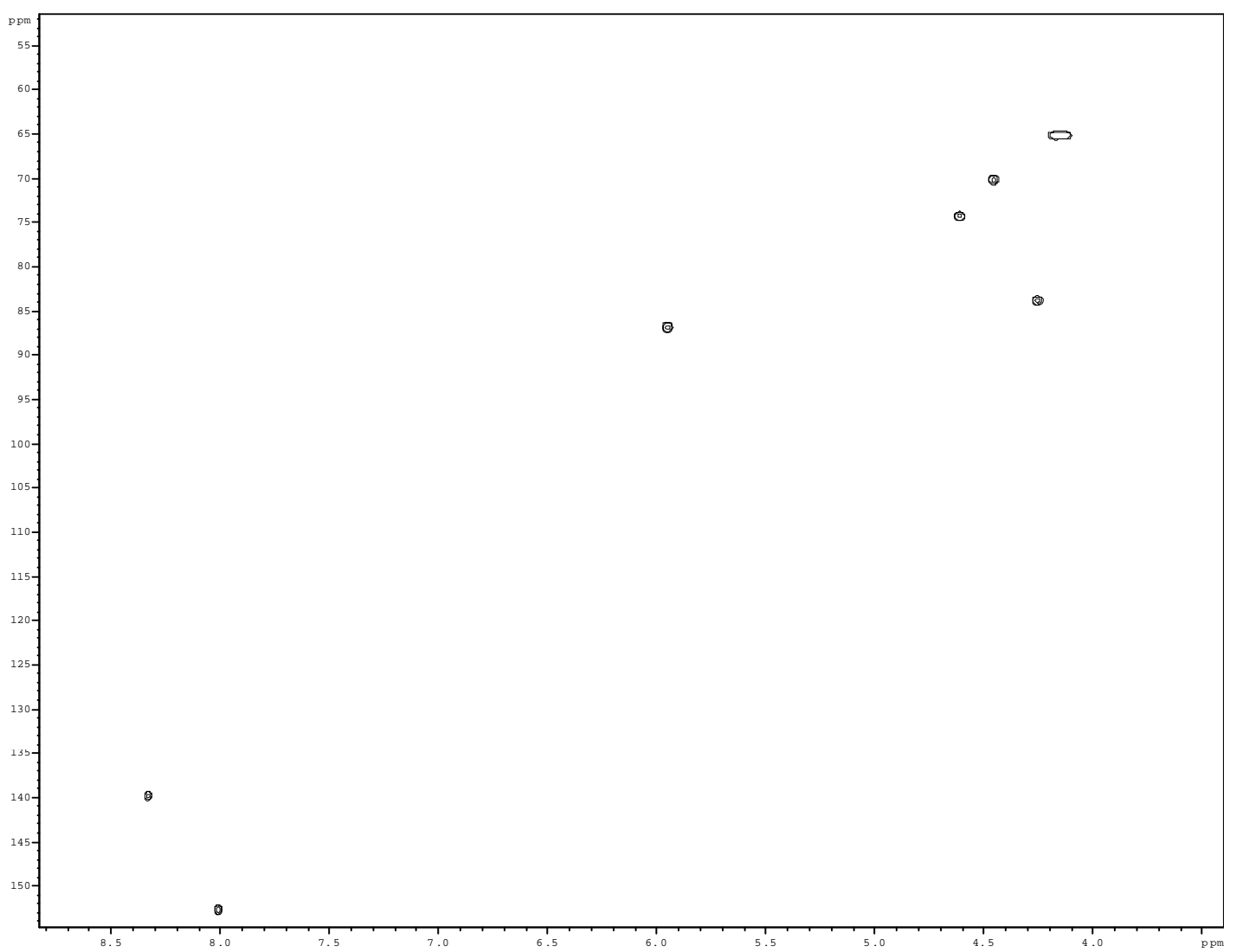




\section{Adenosine 5'- $\beta, \gamma$-fluoromethylenetriphosphate, AppCHFp (3)}

Phosphoryl monofluoromethylene ADP 1d_carbon_narrow D20 \{C: \nmr_users\} mohamady 12

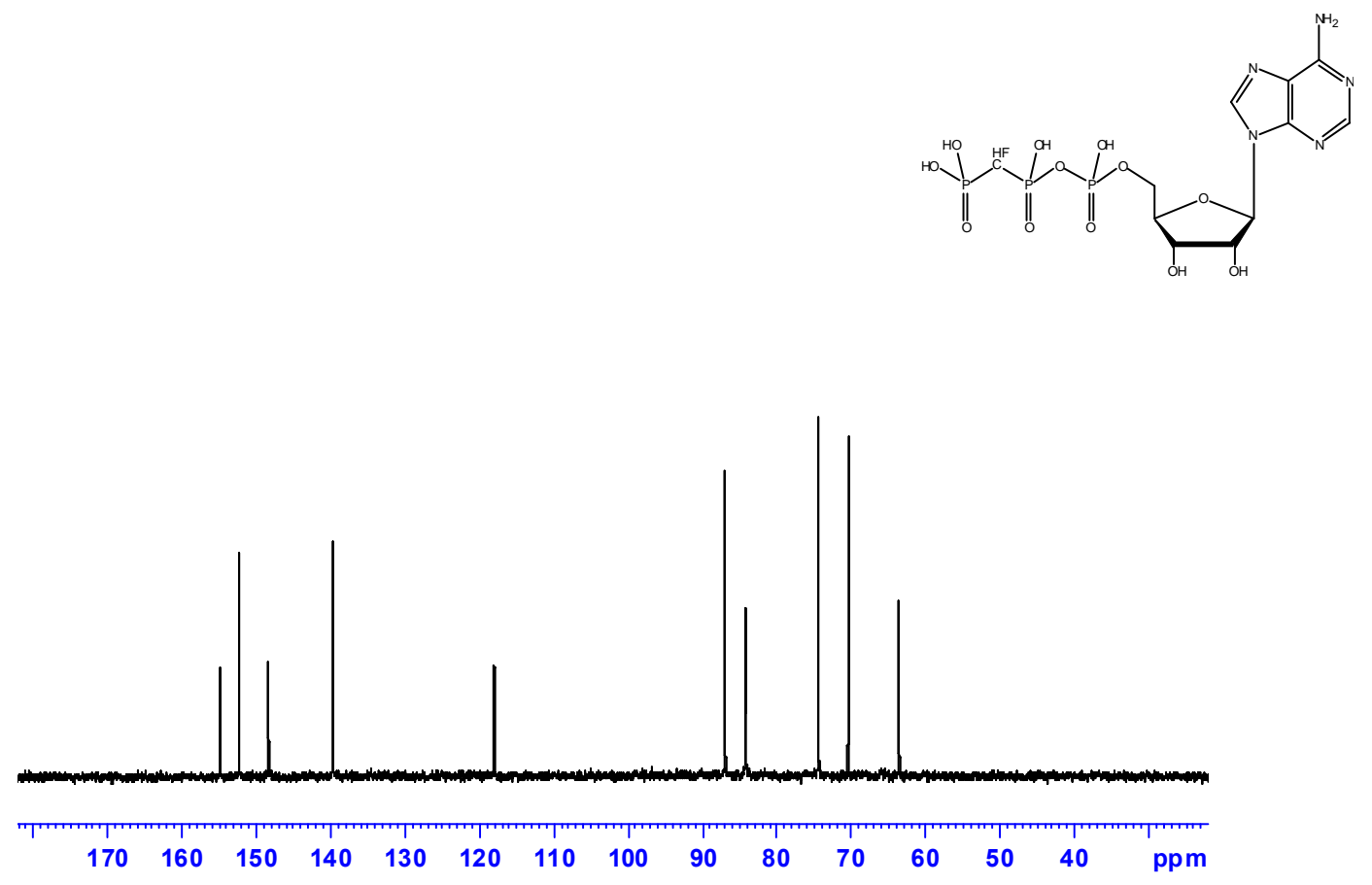


Mono fluoromethyl ADP

1d_proton D20 \{C: \nmr_users\} mohamady 7

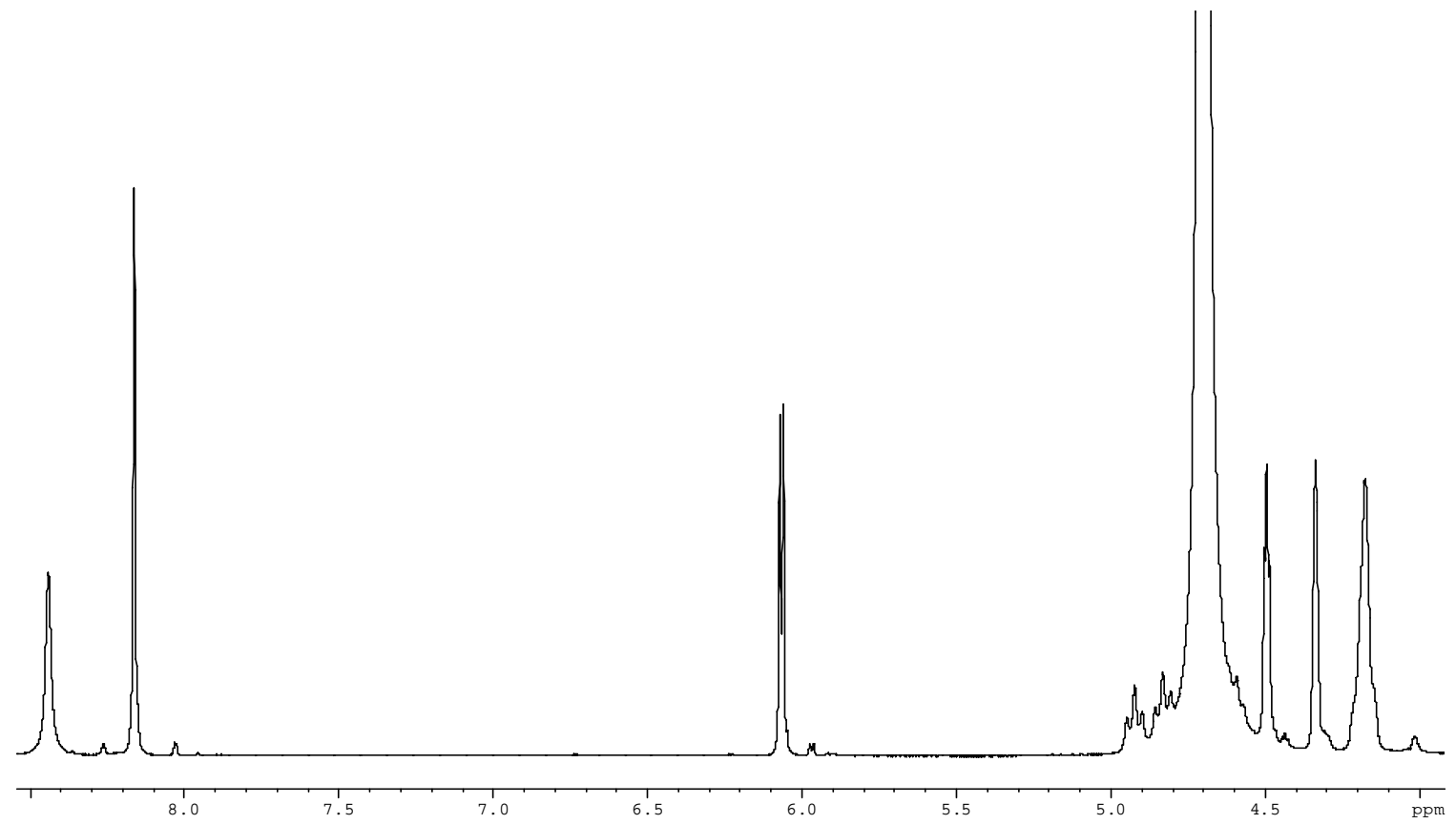




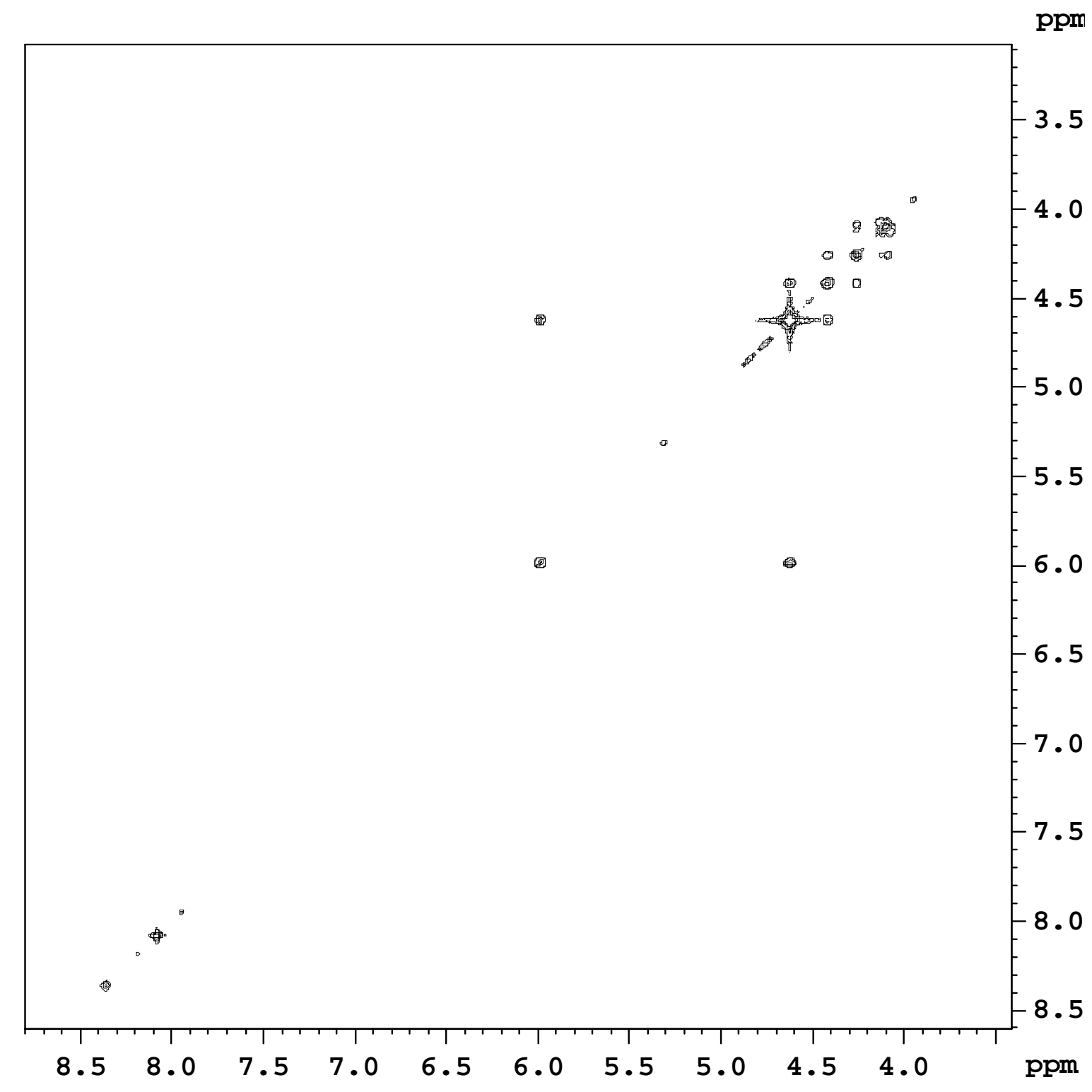


Monofluormethyl ADP

2d_HSQC D20 \{C: \nmr_users $\}$ mohamady 5

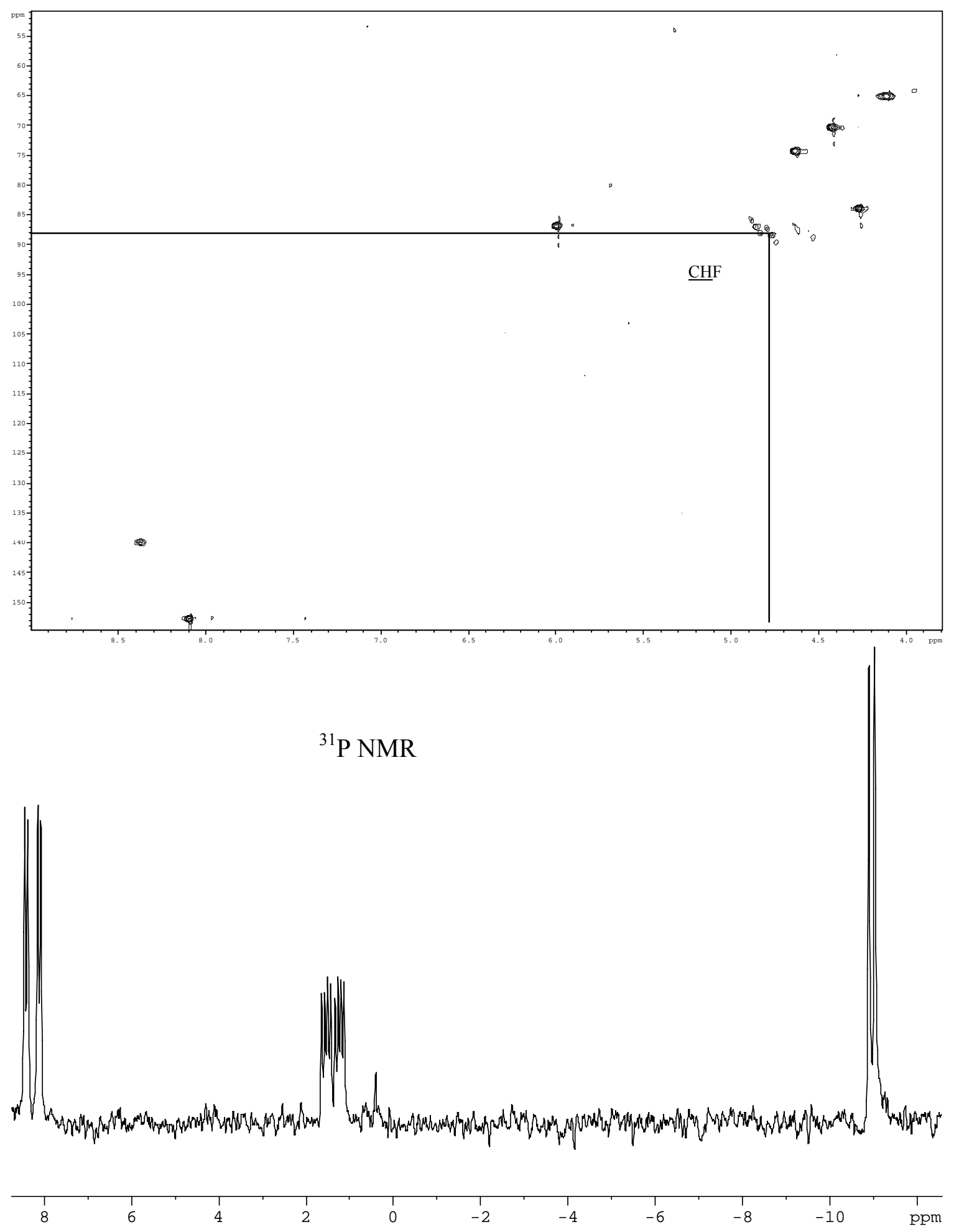


Mohamady and Jakeman

Improved nucleoside triphosphate analogue synthesis

Adenosine 5'- $\beta, Y$-difluoromethylenetriphosphate, $A C_{2} F_{2} p(4)$

Phosphoryldifluoromethyl ADP
1d_carbon_narrow D20 $\{$ C: $\backslash$ nmr_users $\}$ mohamady 15

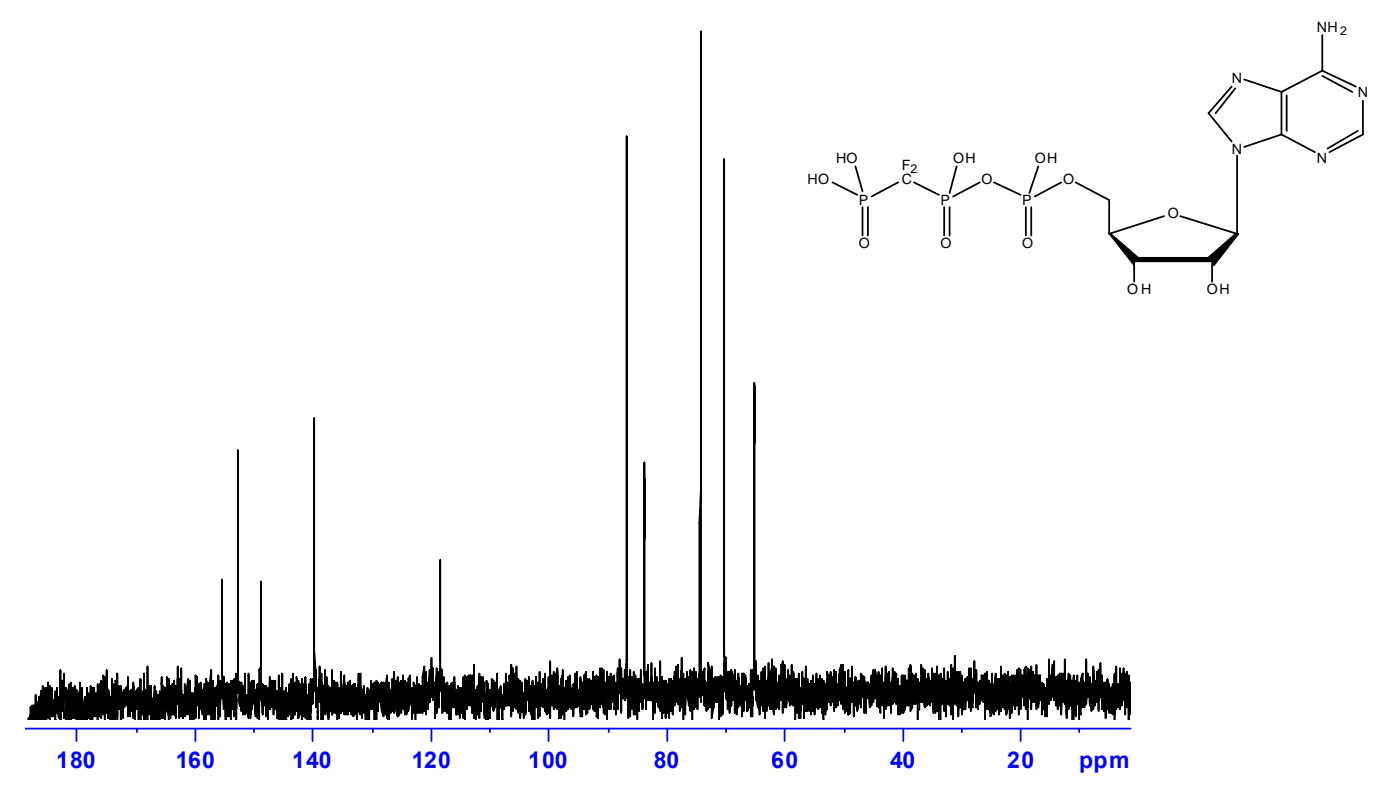

${ }^{19} \mathrm{~F}$ decoupled ${ }^{13} \mathrm{C}$

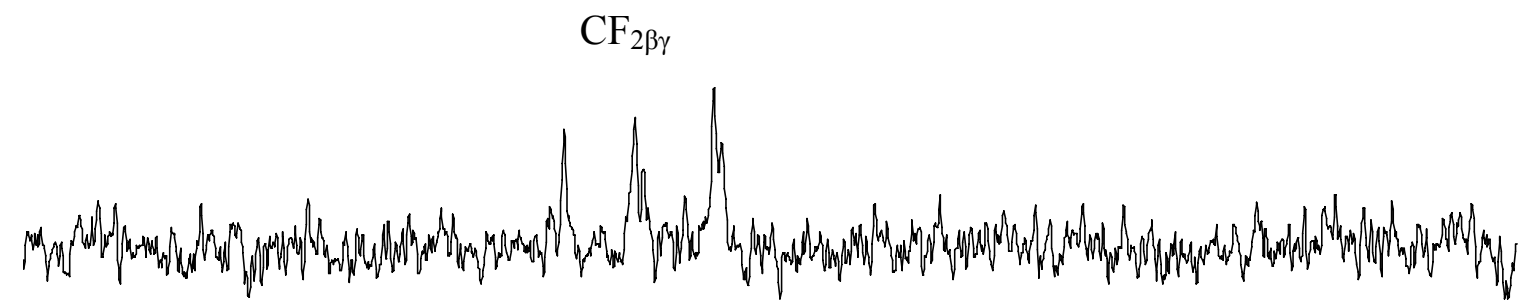

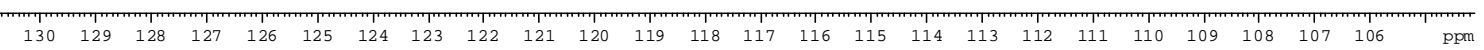

S29 
Phosphoryldifluoromethyl ADP
1d_proton D20 \{C: \nmr_users\} mohamady 15

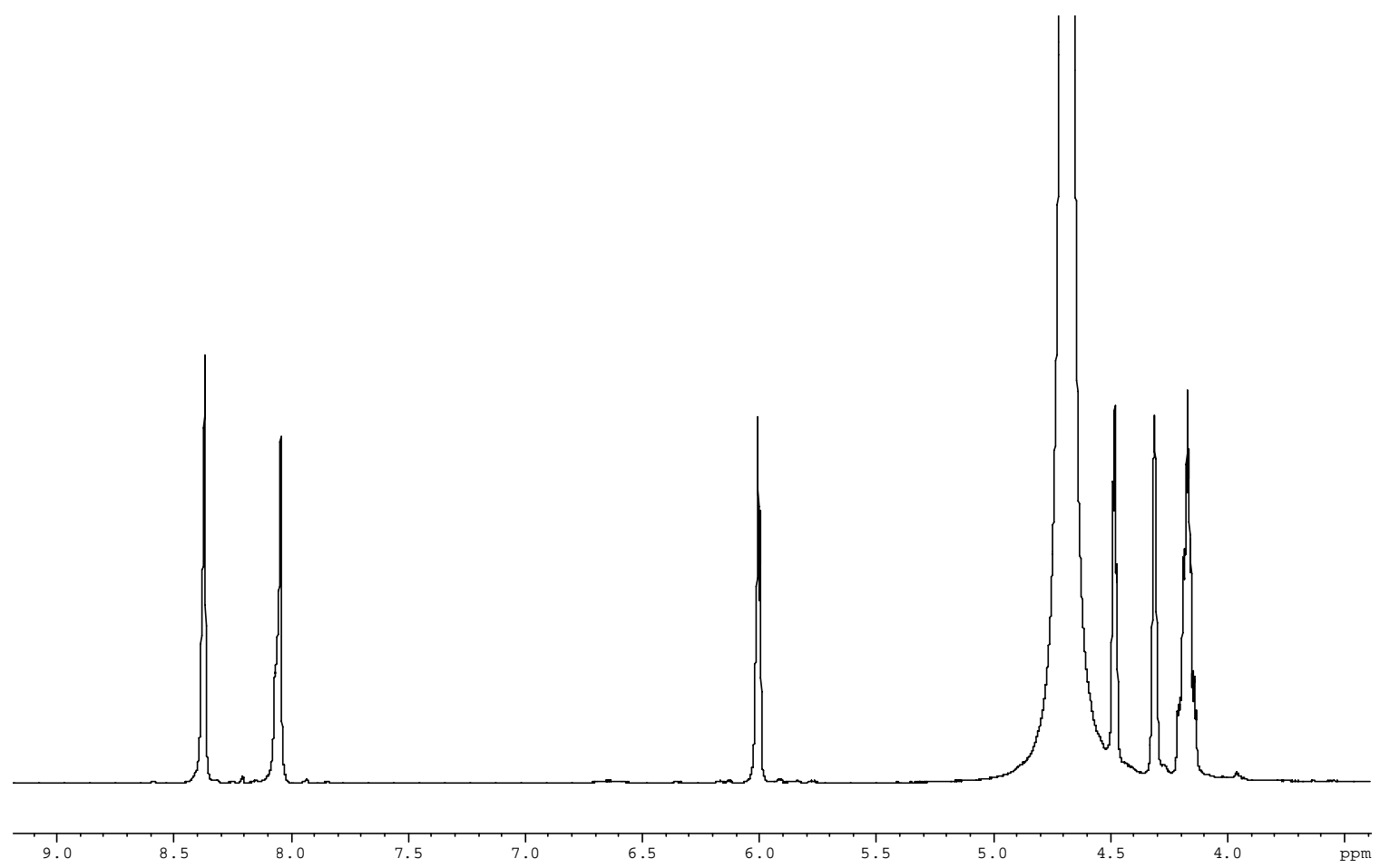




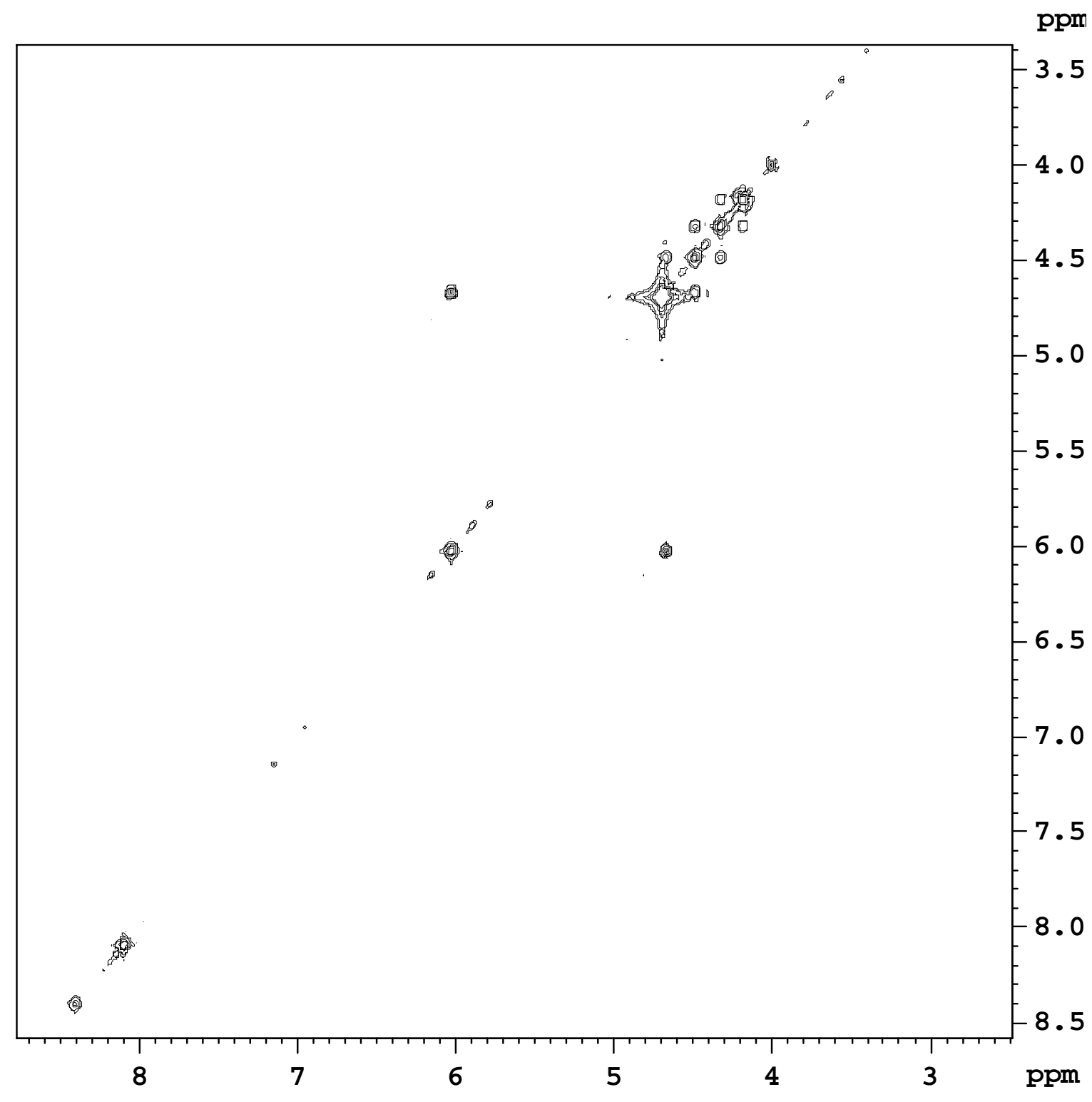



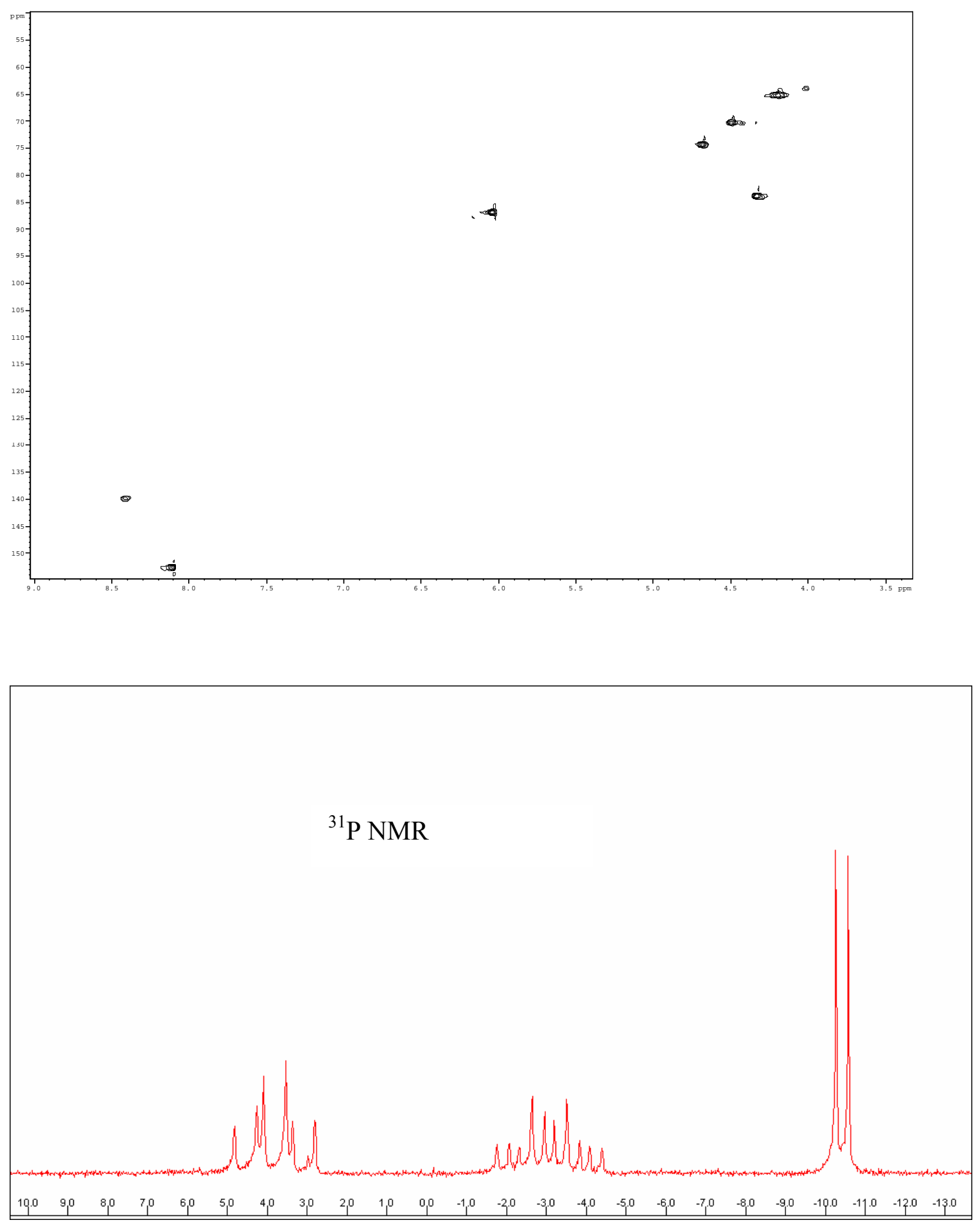


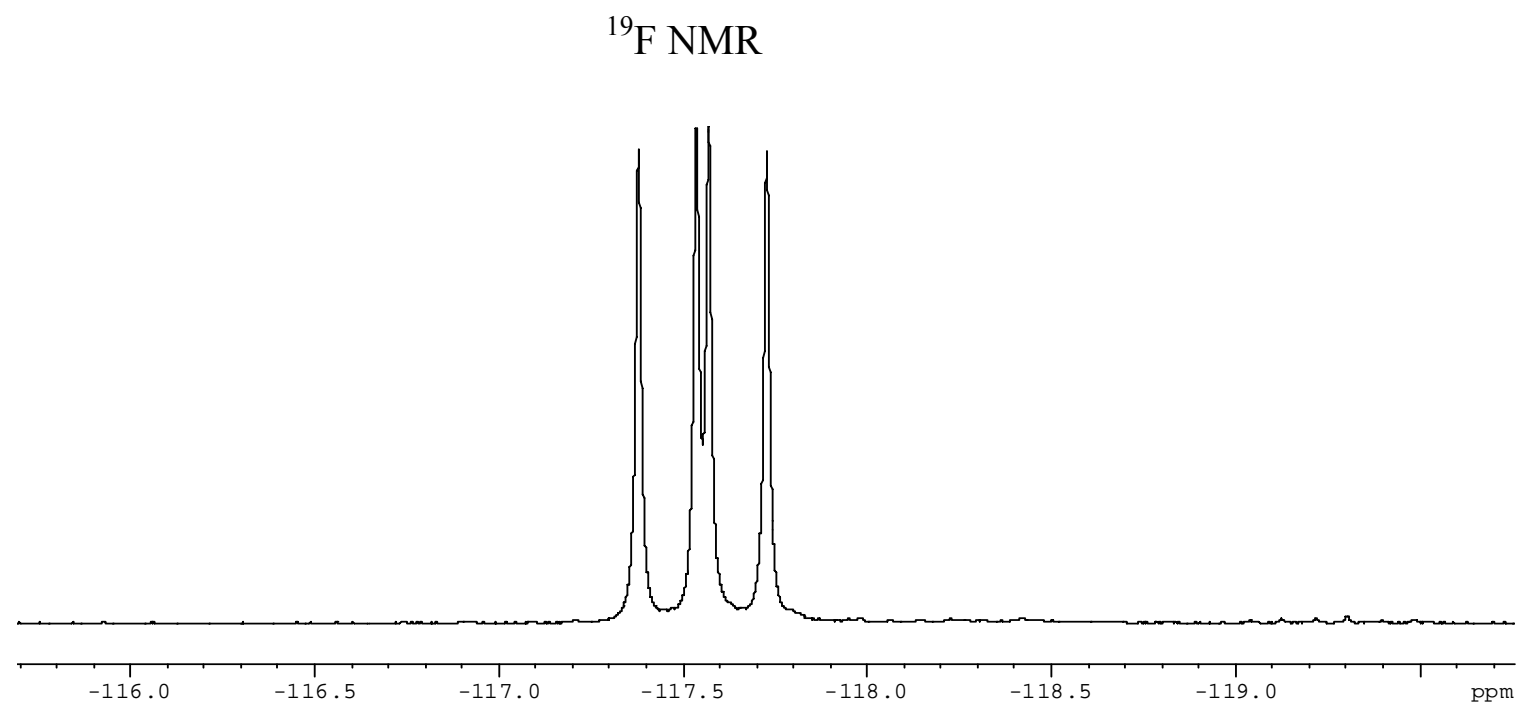




\section{Uridine 5'- $\beta, \gamma-$ methylenetriphosphate, $\mathrm{UppCH}_{2} \mathrm{p}$ (5)
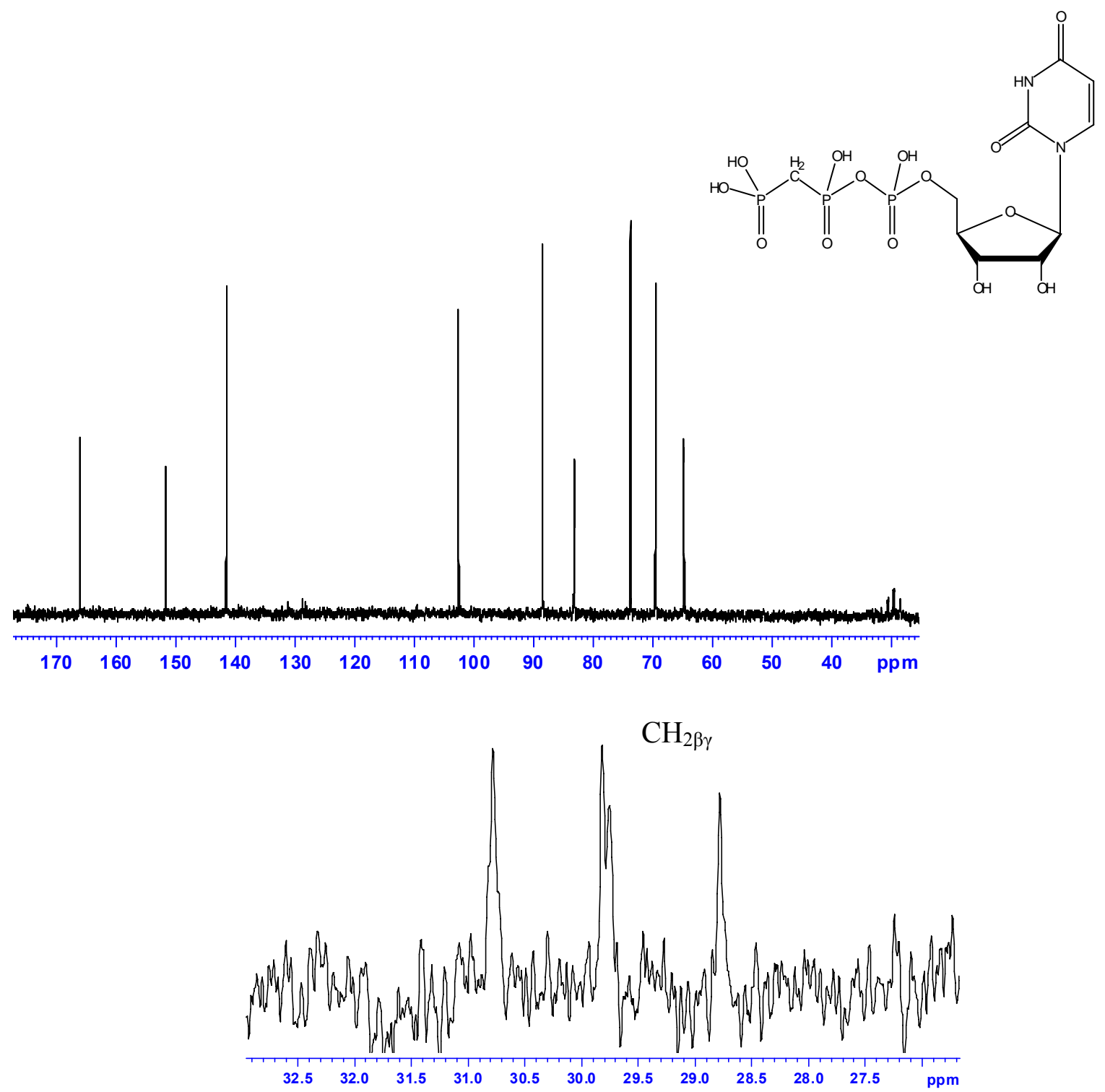


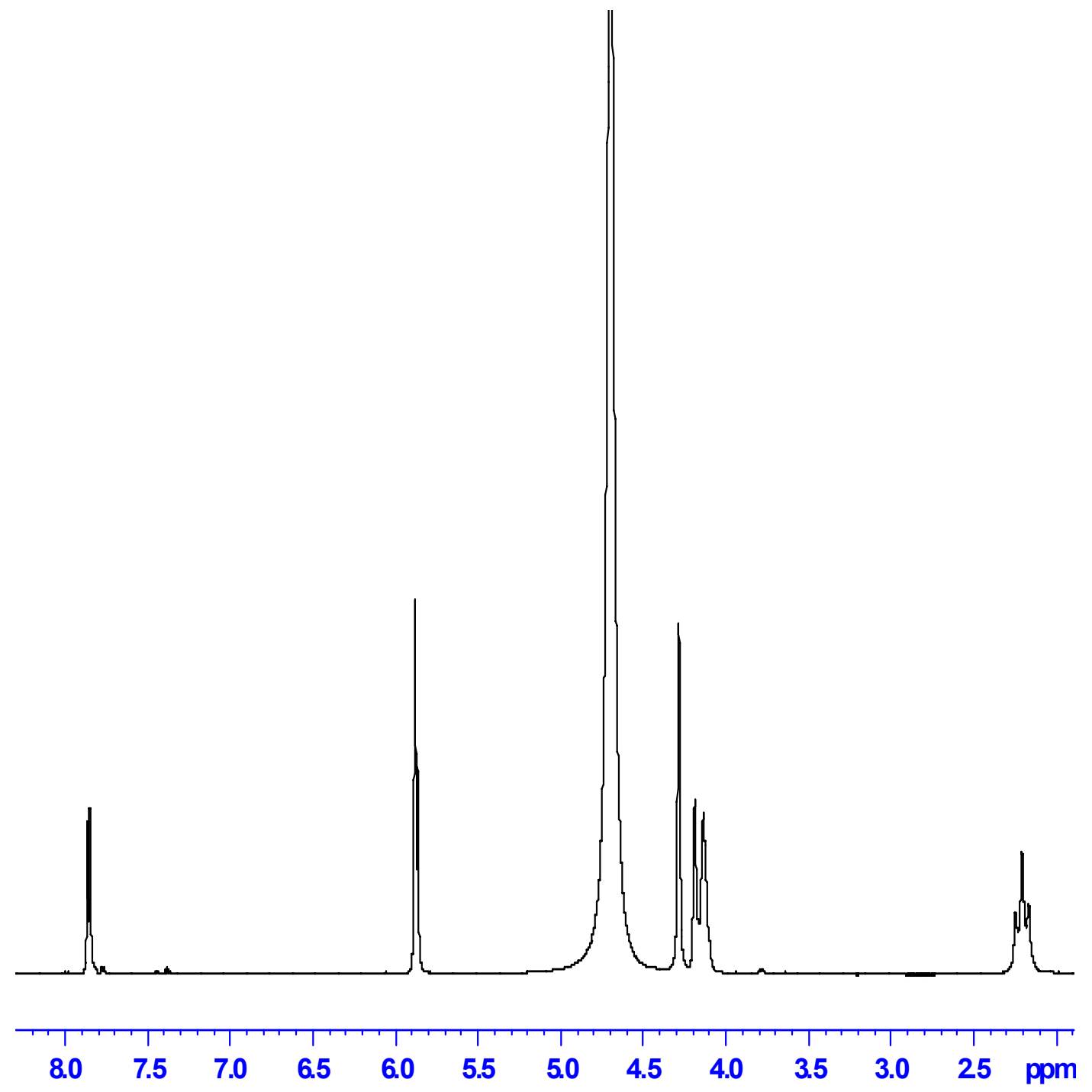


Phosphorylmethyl UDP

2d_HSQC D2O \{C: \nmr_users\} mohamady 16

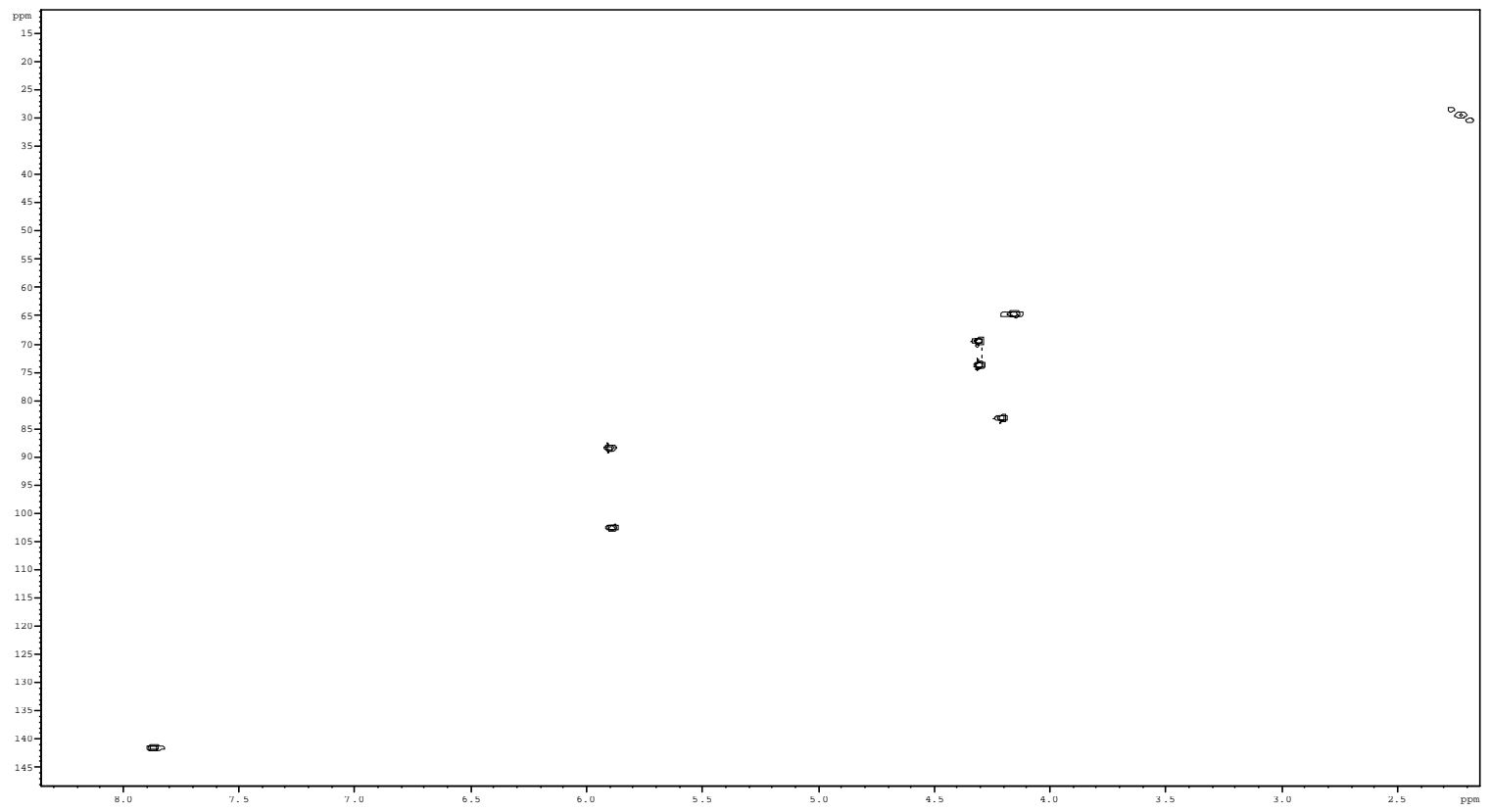




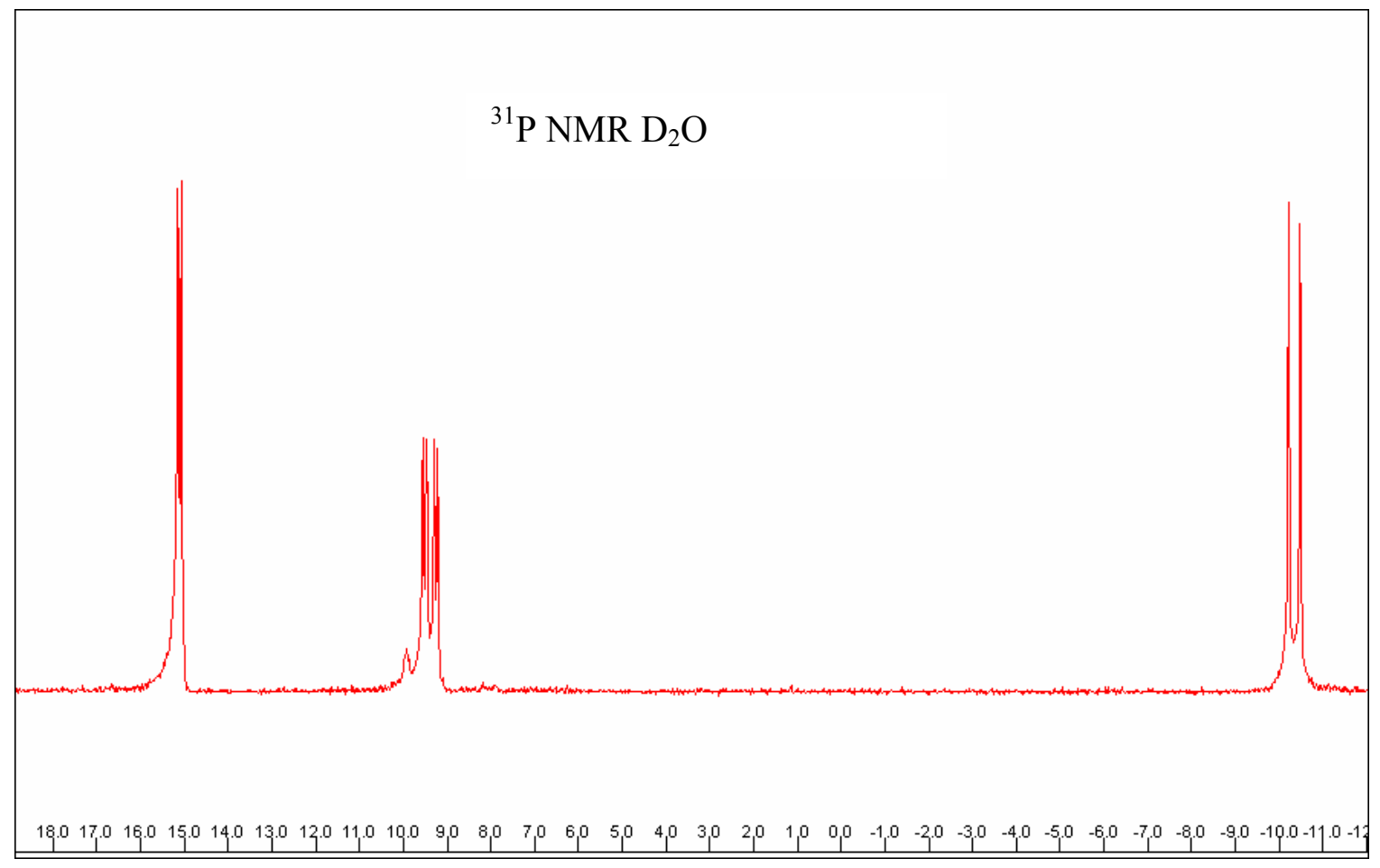




\section{Uridine 5'- $\beta$, $\gamma$-dichloromethylenetriphosphate, $\mathrm{UppCCl}_{2} \mathrm{p}$ (6)}

Phosphoryl dichloromethyl UDP
1d_carbon_wide D20 $\{\mathrm{C}: \backslash \mathrm{nmr}$ _users $\}$ mohamady 8
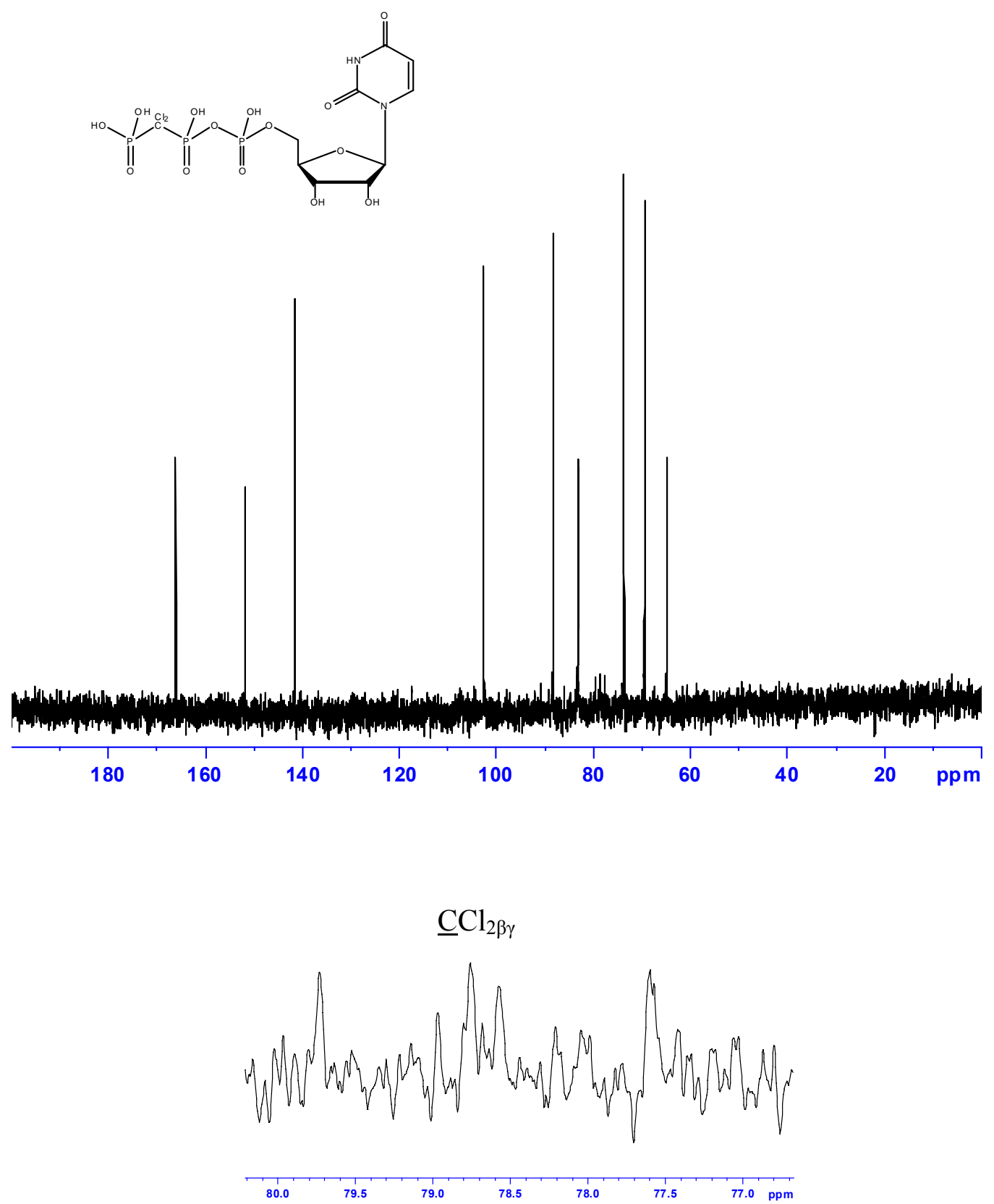


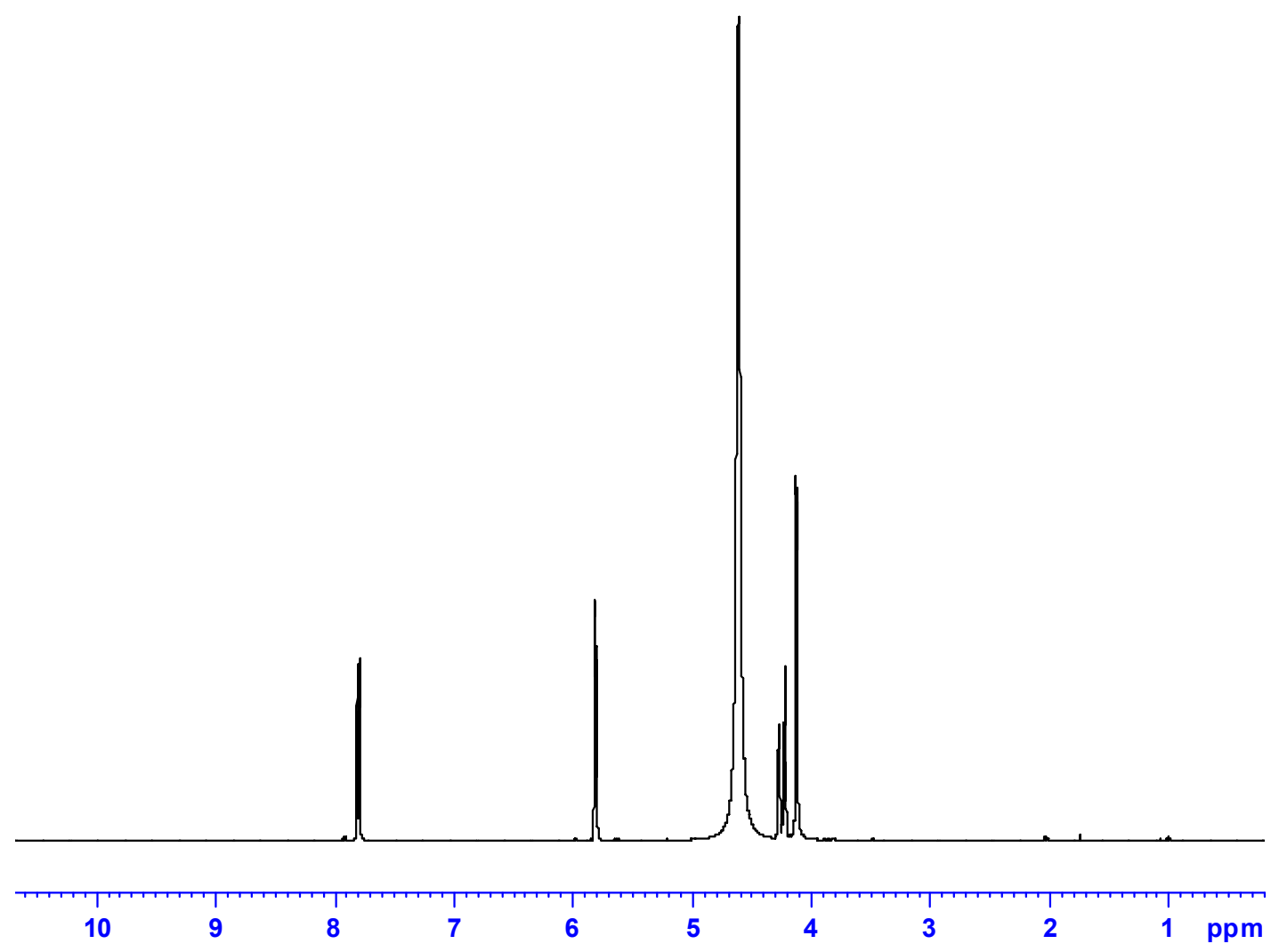




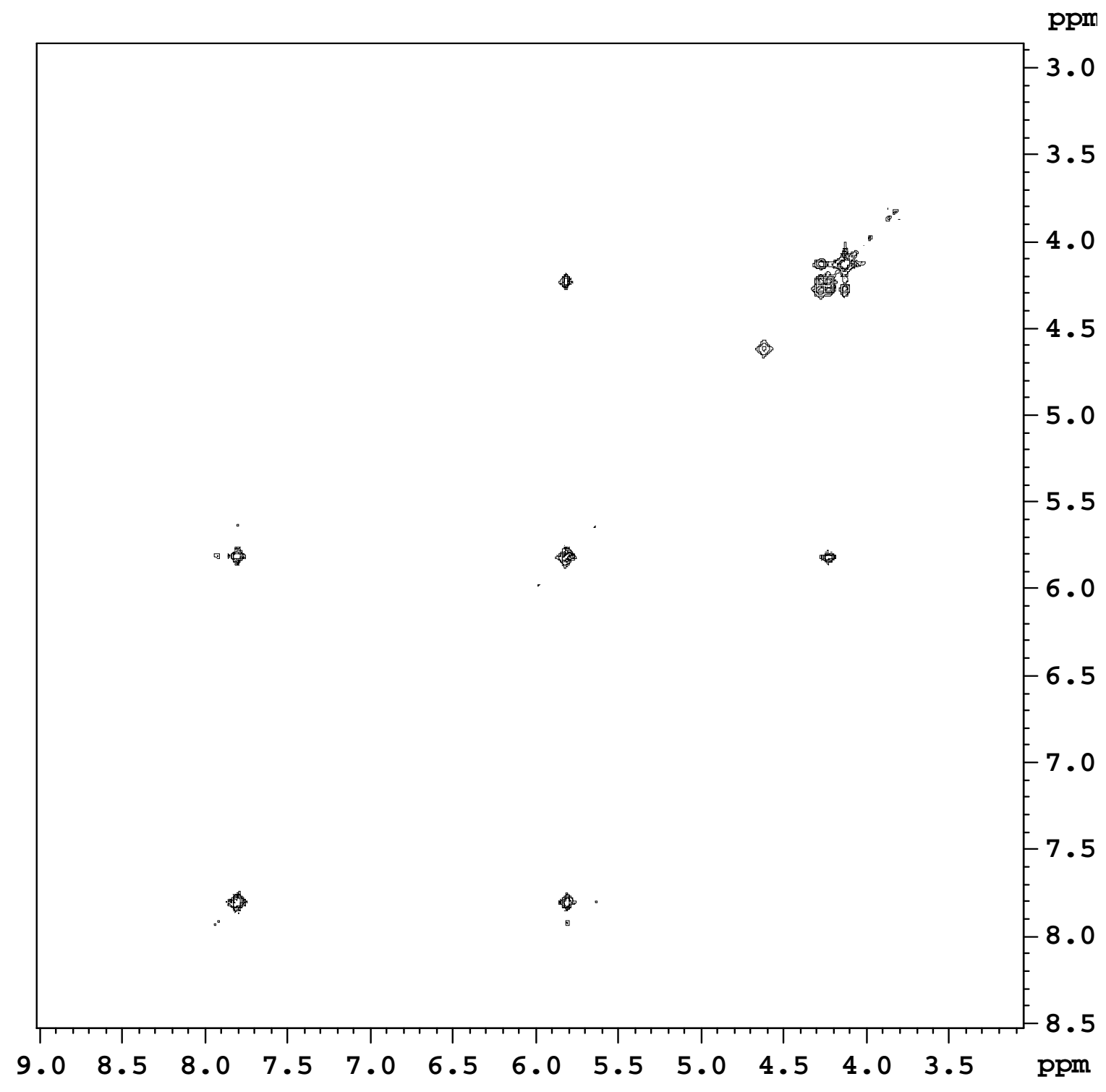


Phosphoryl dichloromethyl UDP

2d_HSQC D20 \{C: \nmr_users\} mohamady 8
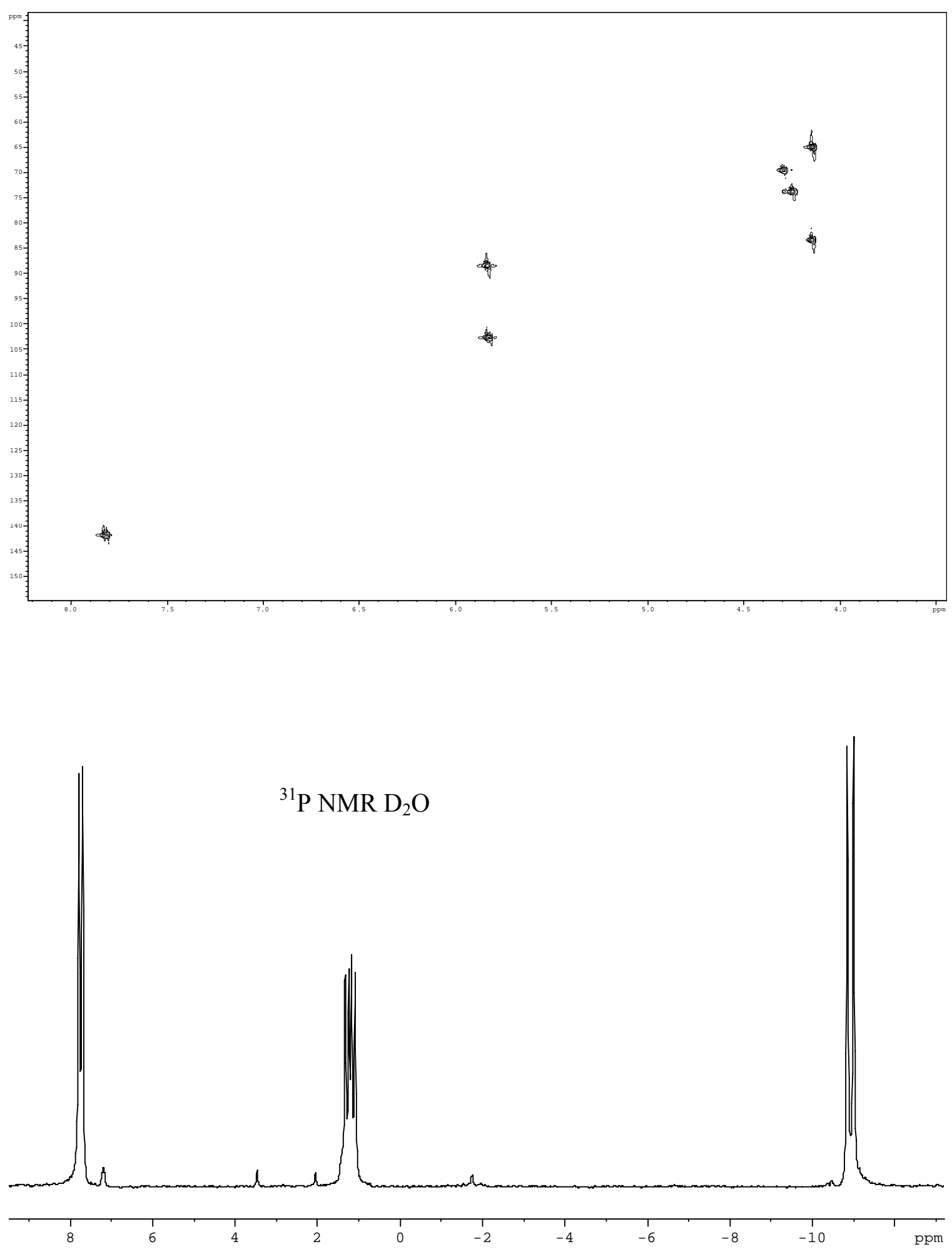
Mohamady and Jakeman

Improved nucleoside triphosphate analogue synthesis

Uridine 5'- $\beta, \gamma$-fluoromethylenetriphosphate, UppCHFp (7)

Phosphoryl monofluorouDP
1d_carbon_wide D20 \{C: $\backslash$ nmr_users $\}$ mohamady 22

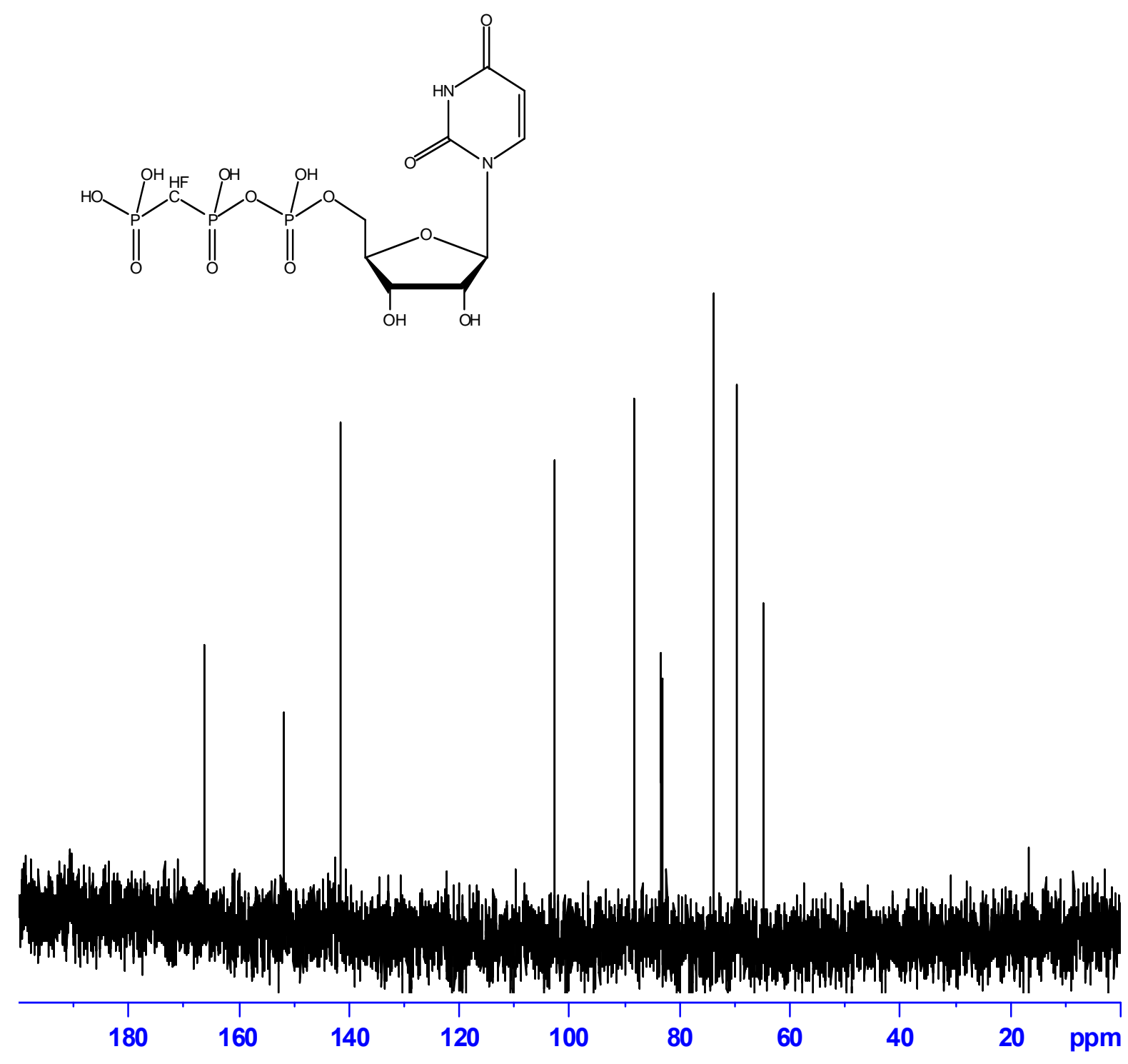

S42 
Phosphoryl monofluoromethyl UDP

2d_COSY D20 \{C: \nmr_users\} mohamady 8

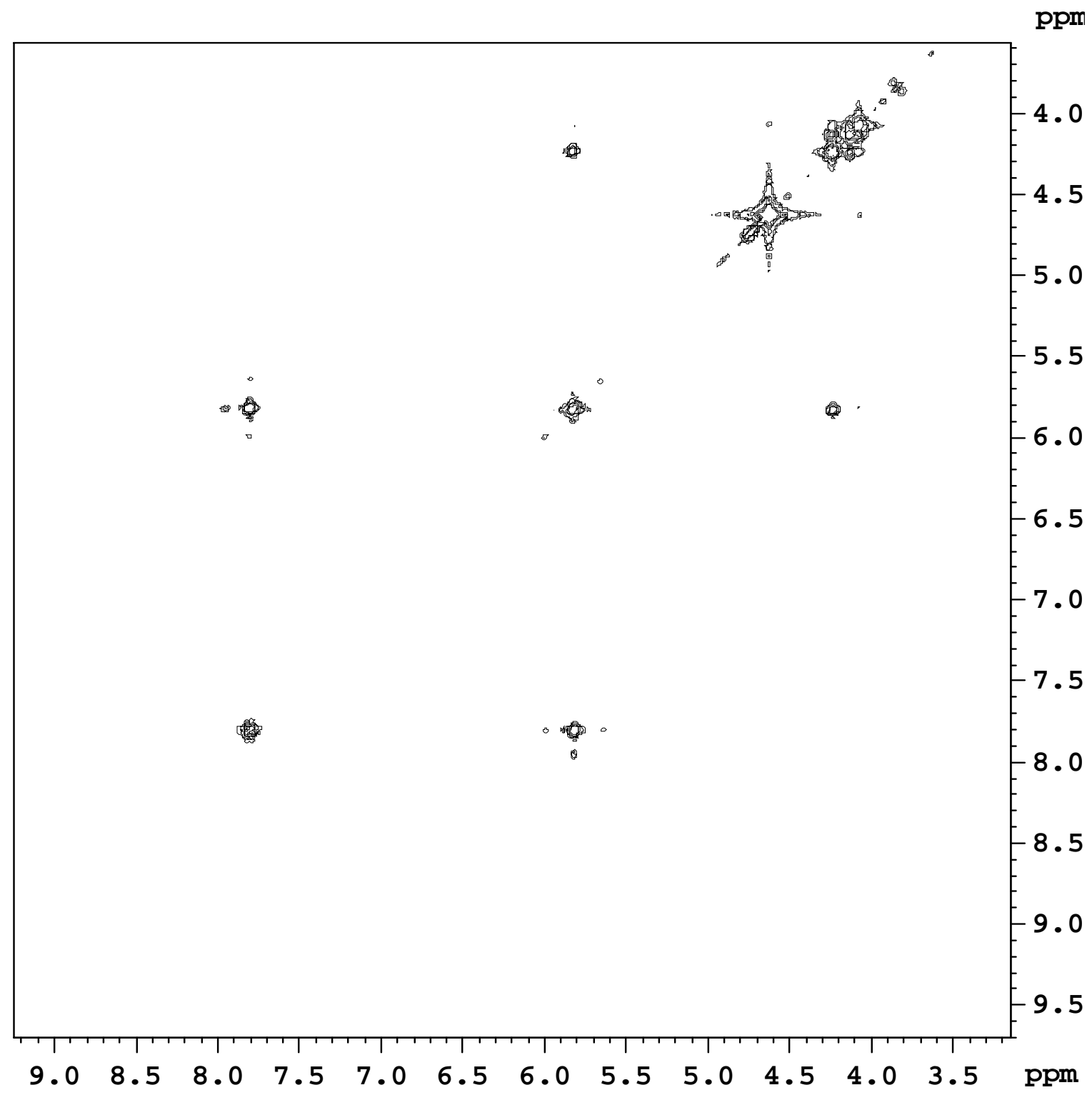


Phosphoryl monofluoromethyl UDP

2d_HSQC D20 \{C: \nmr_users\} mohamady 8

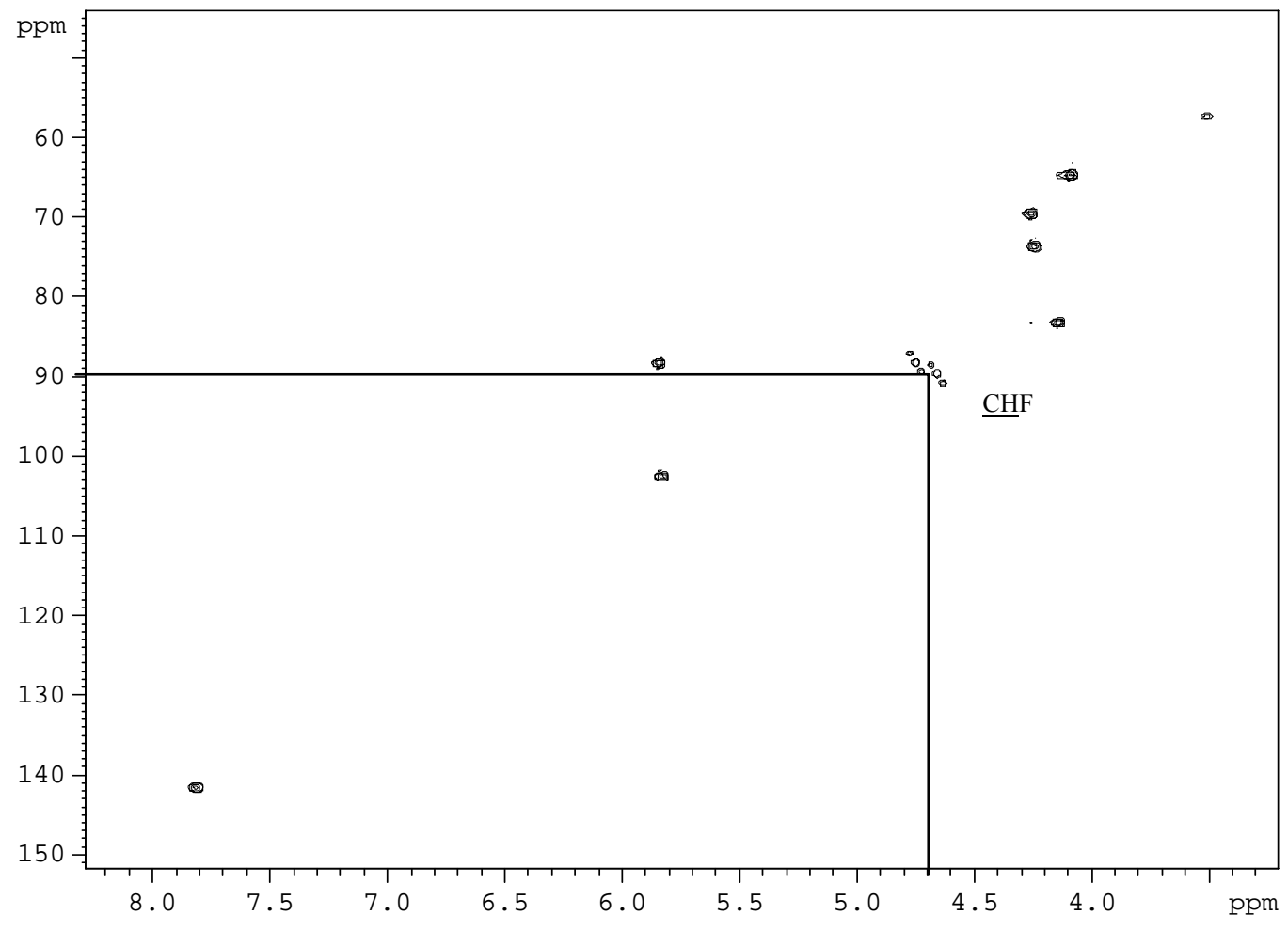


Phosphoryl monofluoroUDP

1d_proton D2O \{C: \nmr_users $\}$ mohamady 22

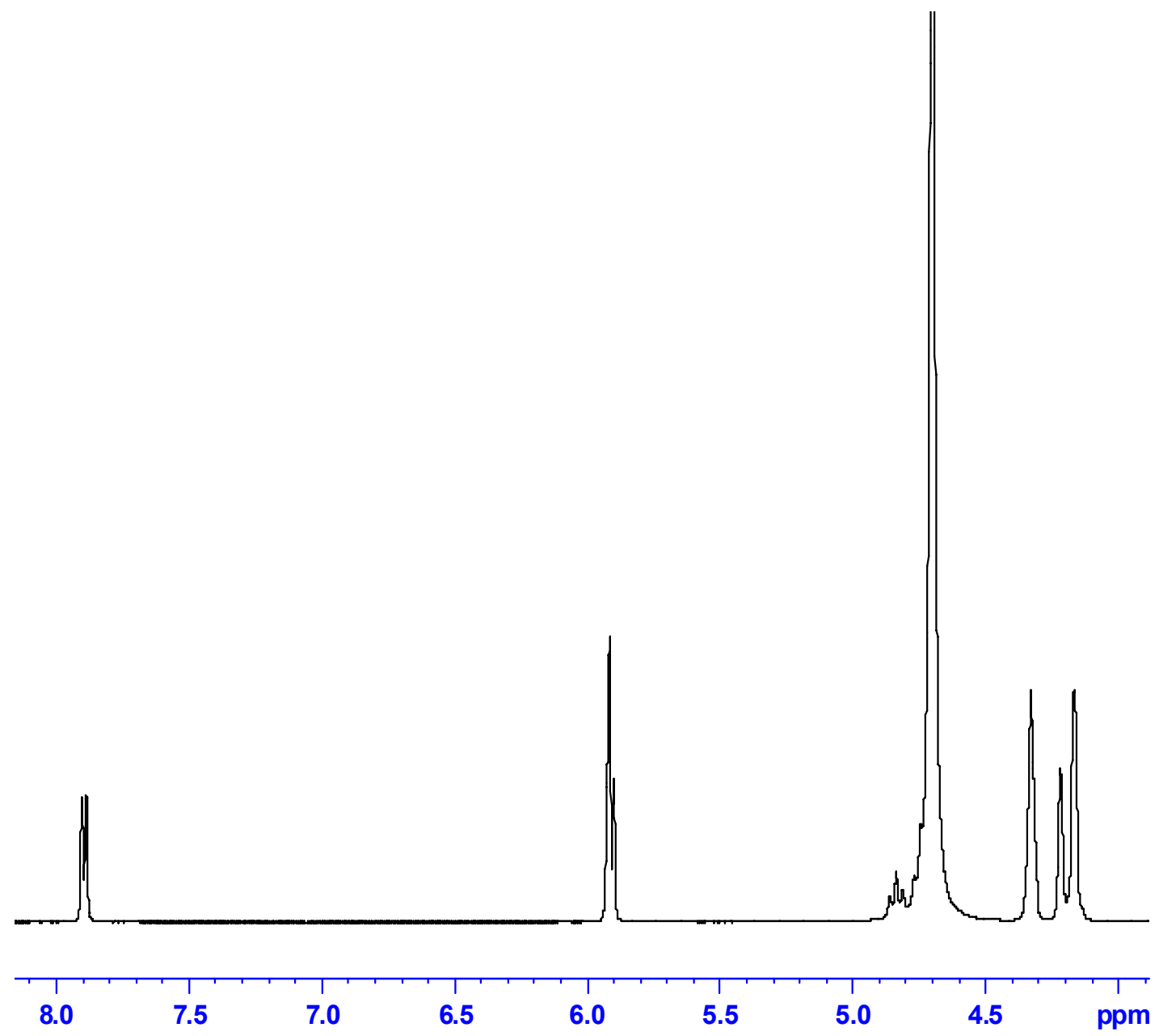




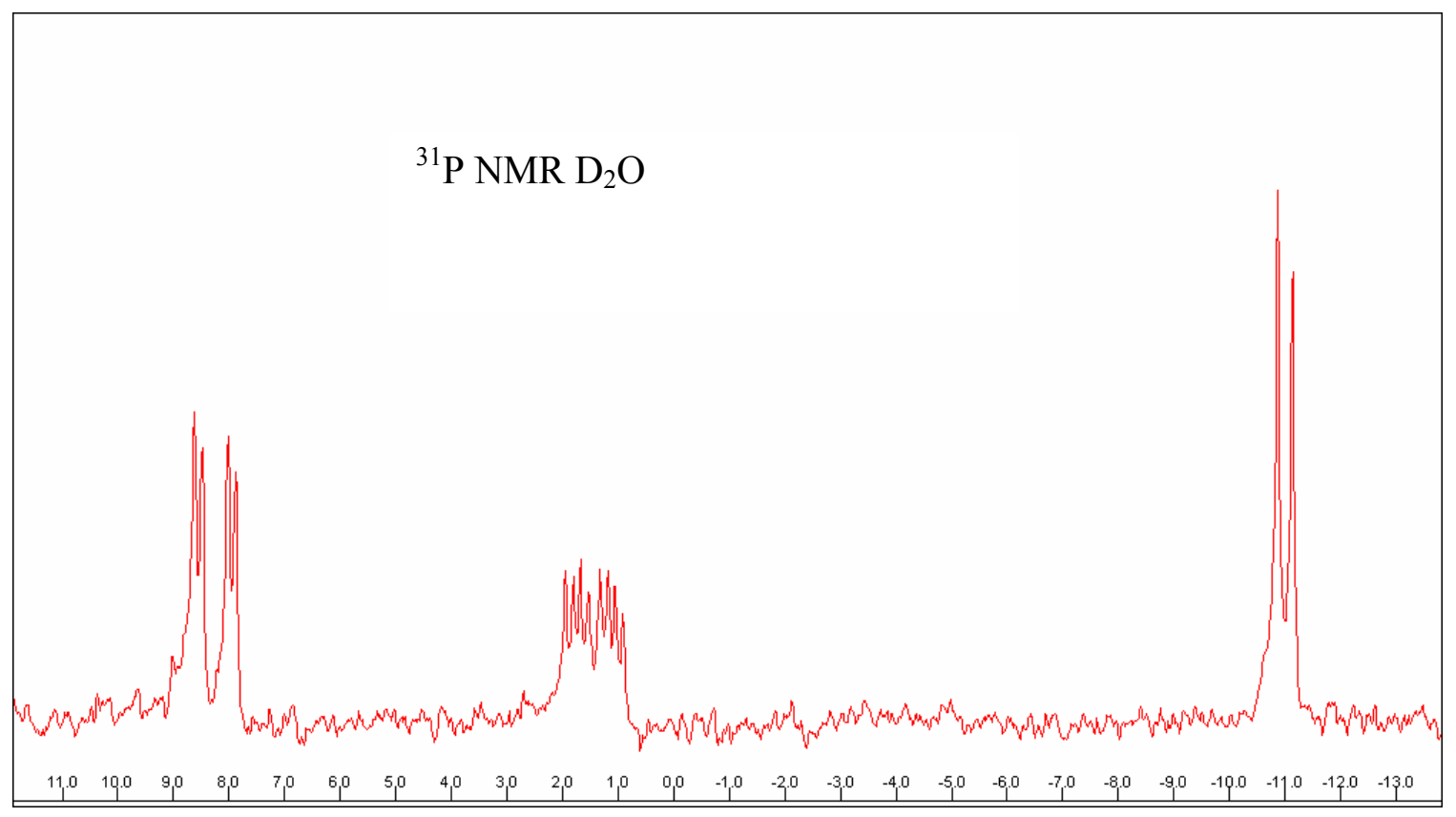




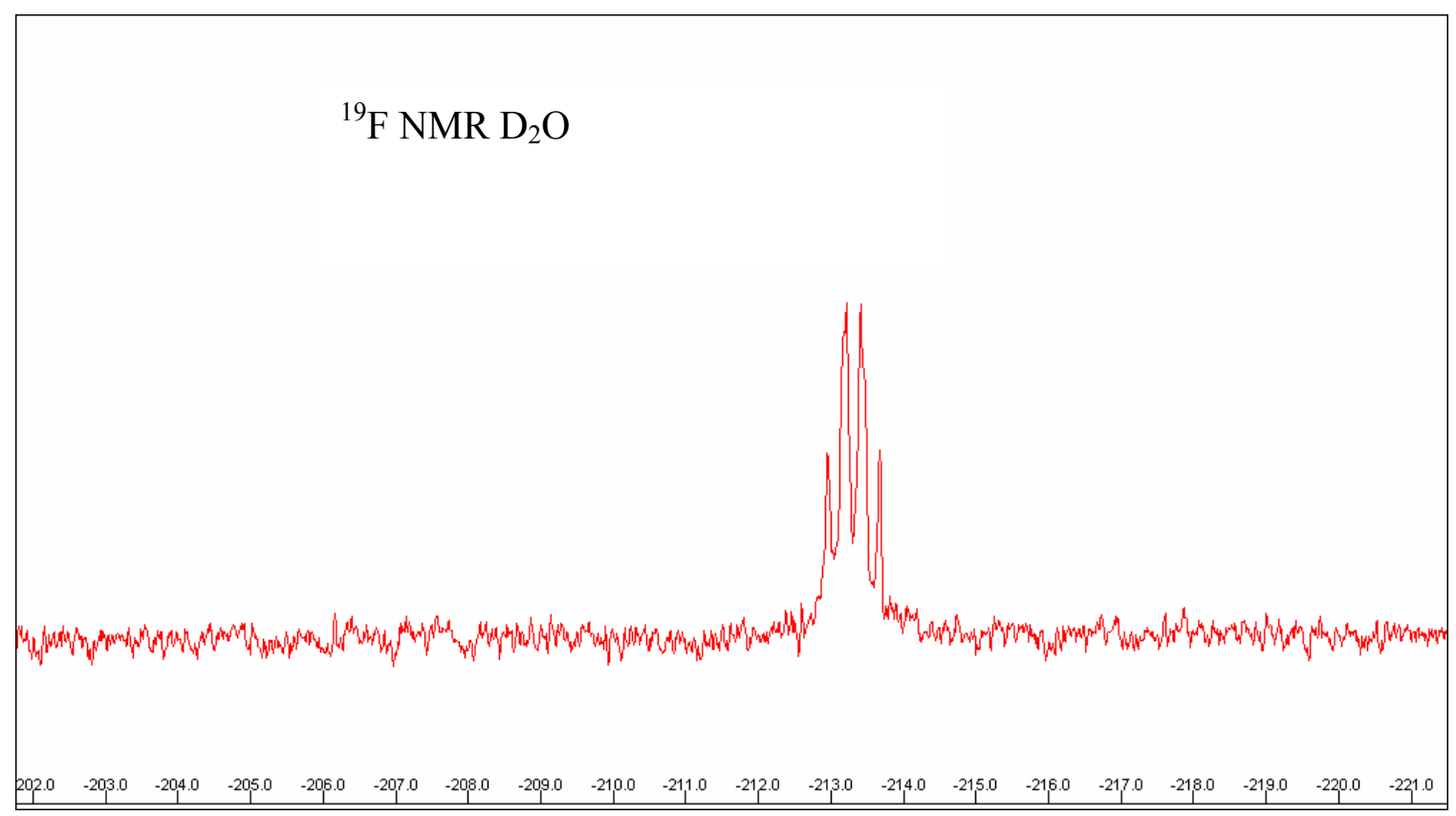




\section{Uridine 5'- $\beta, \gamma$-difluoromethylenetriphosphate, UppCF $2 p(8)$}

Phosphoryl difluoromethyl UDp
1d_carbon_wide D20
$\{$ C: $\backslash$ nmr_users $\}$ mohamady 23

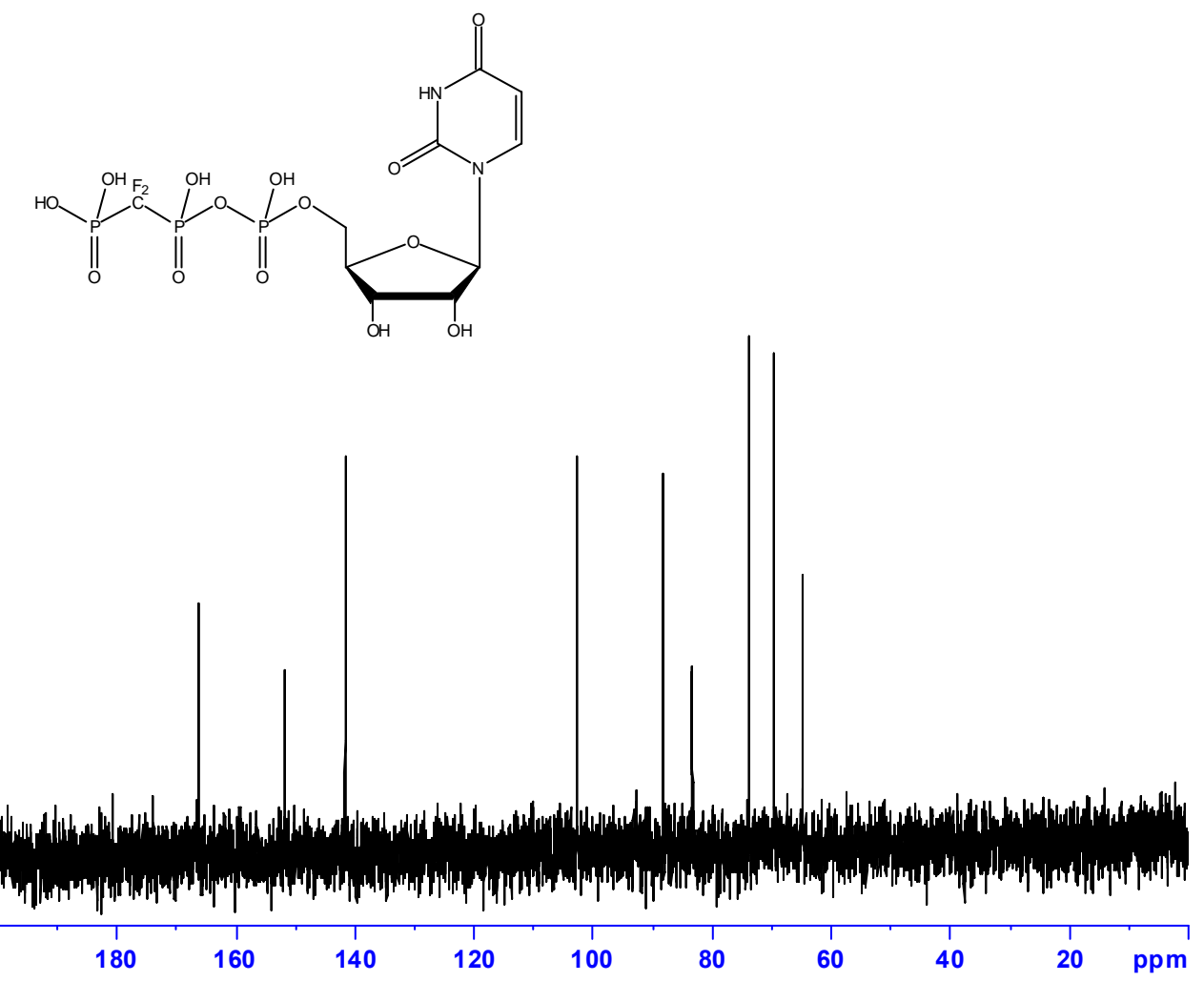

${ }^{19} \mathrm{~F}$ decoupled ${ }^{13} \mathrm{C}$

$\mathrm{CF}_{2} \beta \gamma$

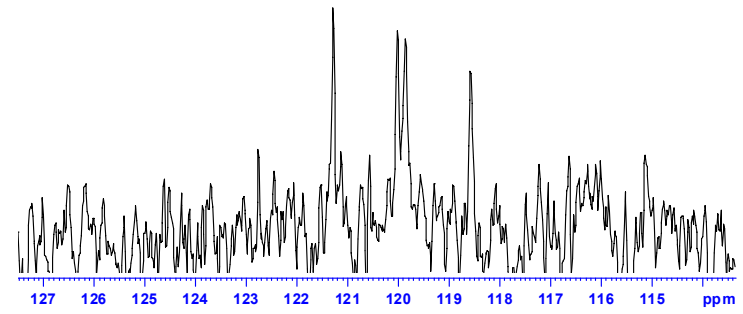



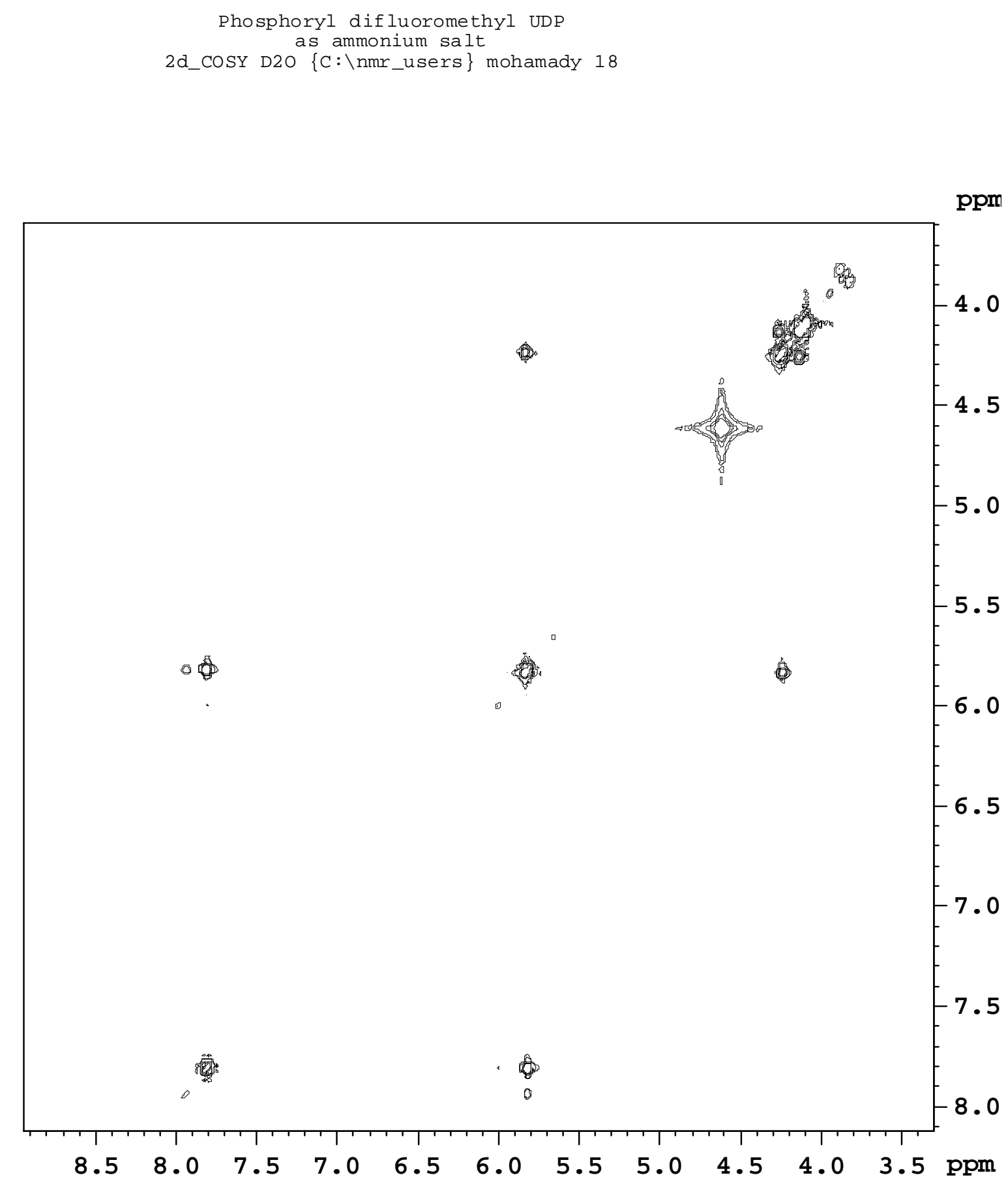
Phosphoryl difluoromethyl UDP

2d_HSQC D20 $\{\mathrm{C}: \backslash \mathrm{nmr}$ _users $\}$ mohamady 18

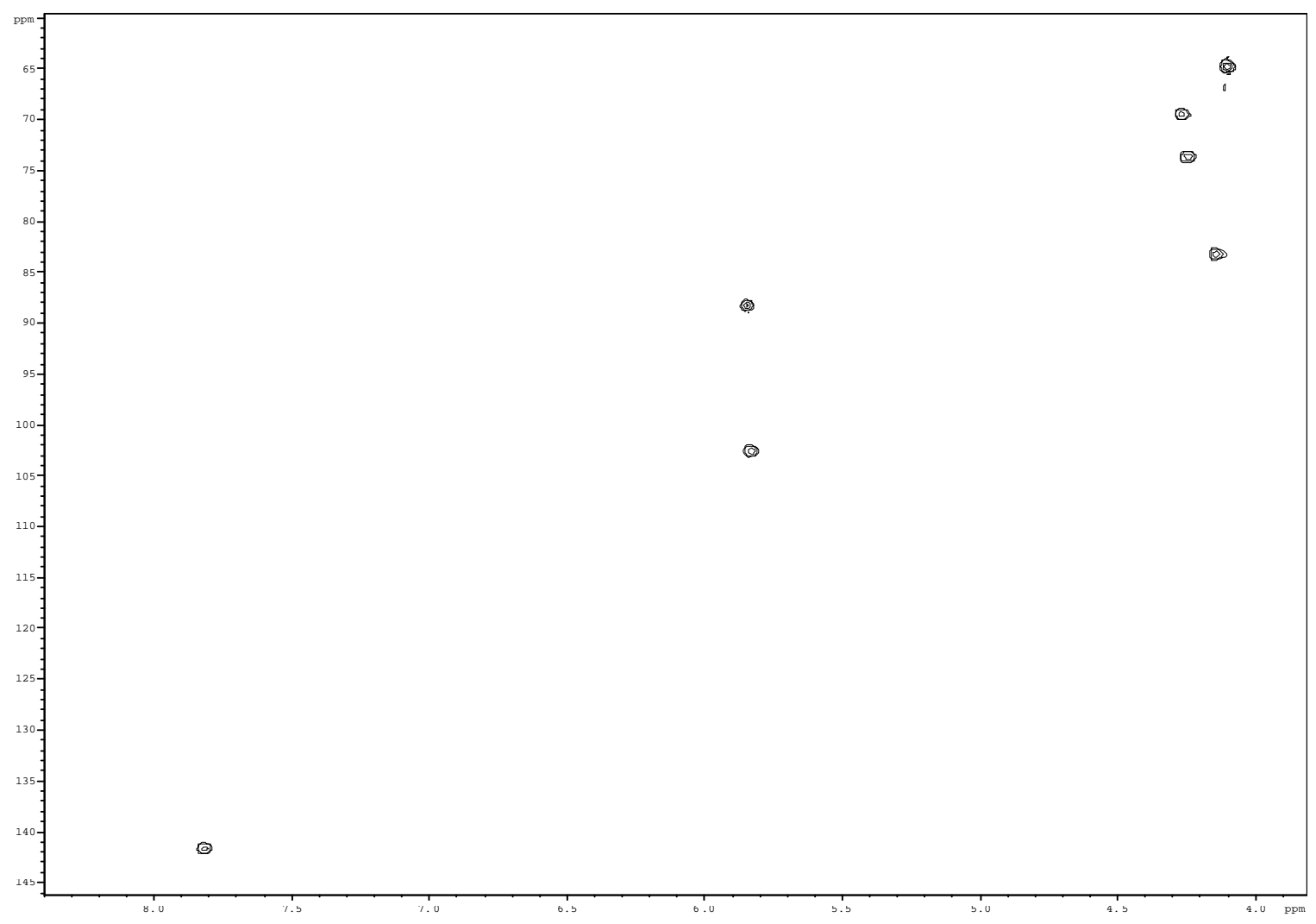




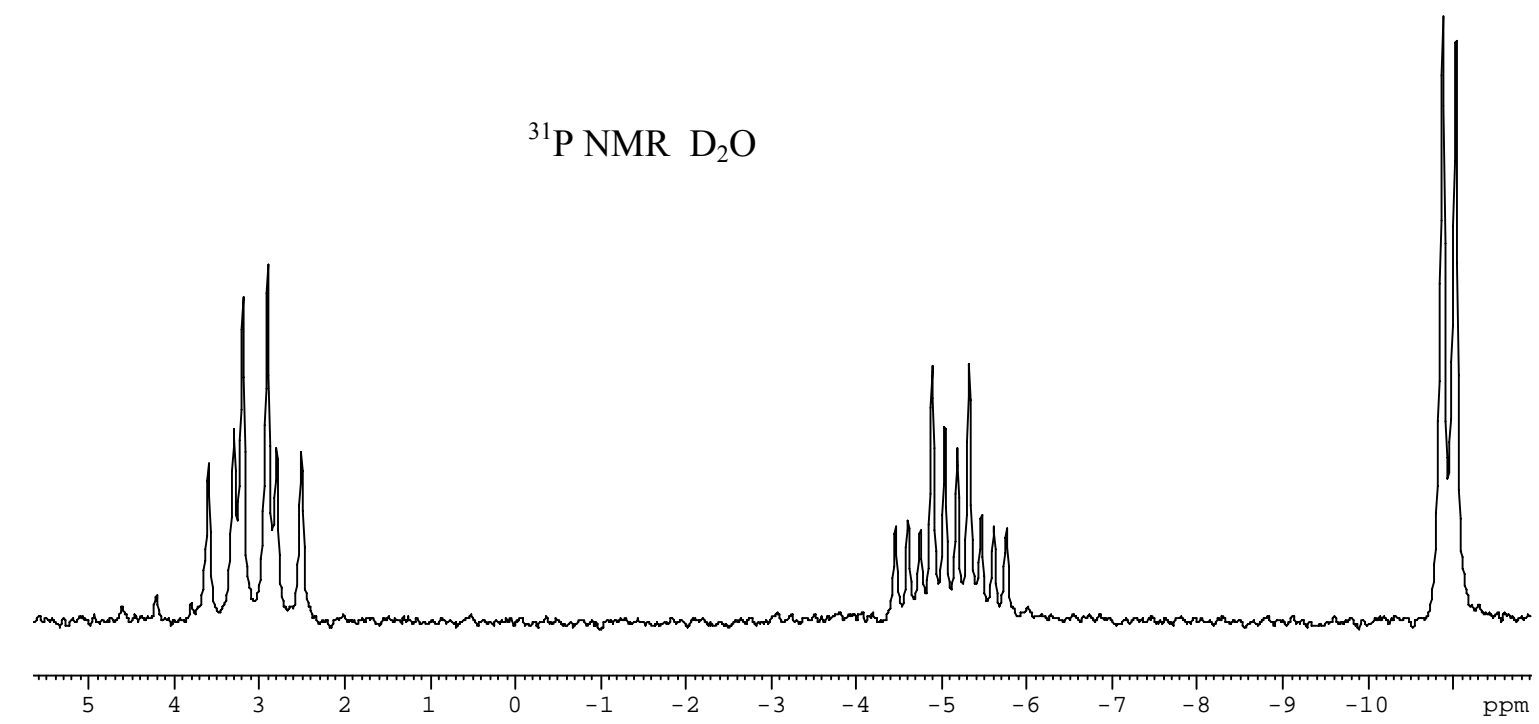

Phosphoryl difluoromethyl UDp
1d_proton D20 \{C: \nmr_users\} mohamady 23

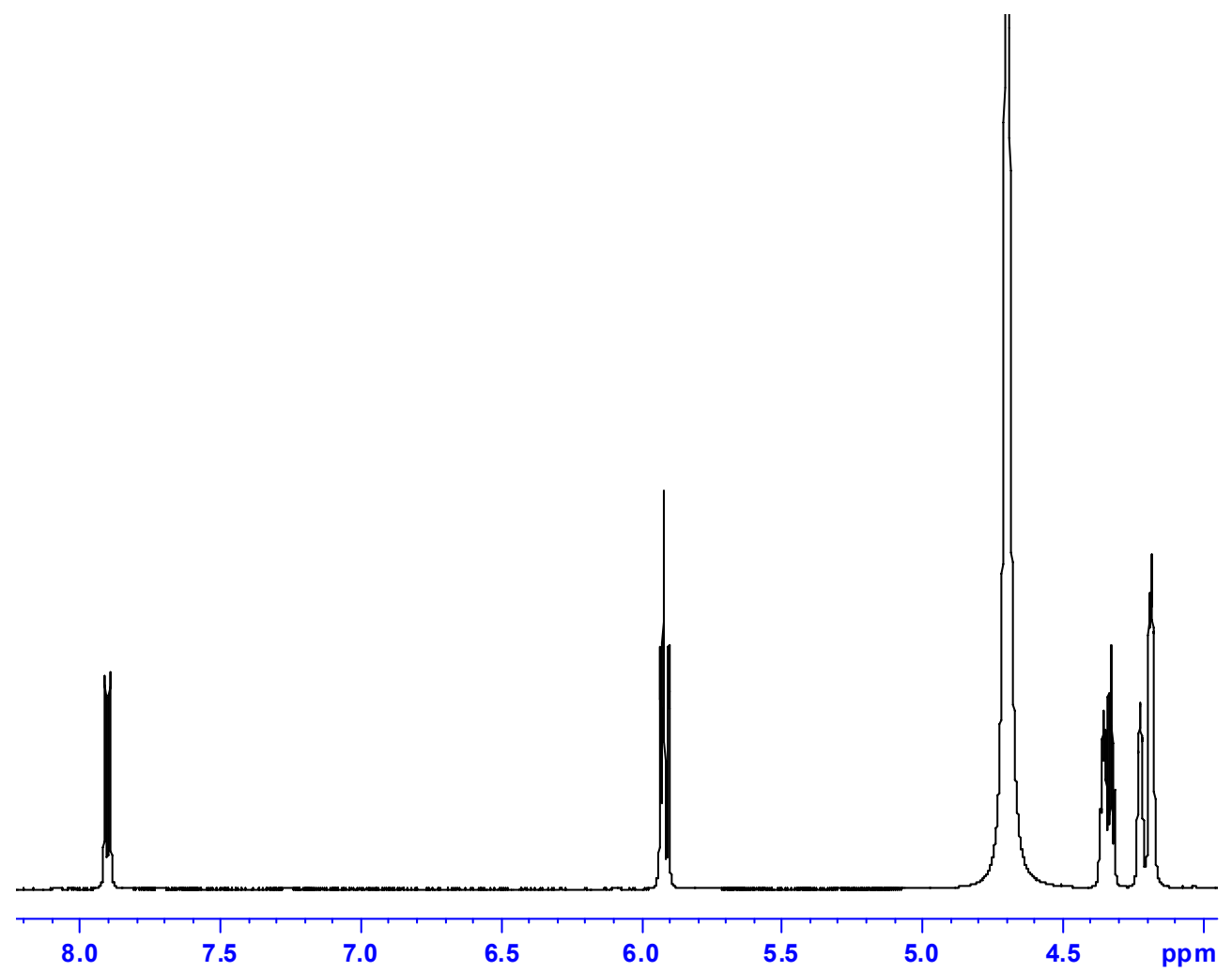




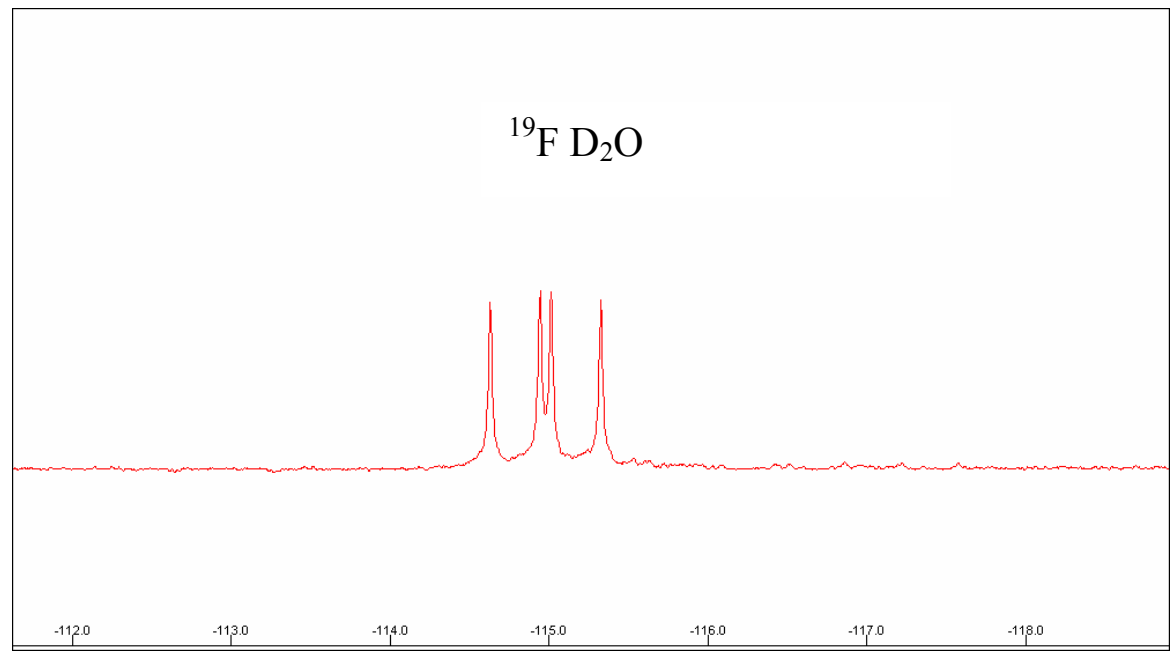

